\title{
Mapping strong electronic coupling in metavalent PbS moiré superlattices
}

Haimei Zheng ( $\nabla$ hmzheng@lbl.gov )

Lawrence Berkeley National Laboratory https://orcid.org/0000-0003-3813-4170

\section{Yu Wang}

Lawrence Berkeley National Laboratory

\section{Zhigang Song}

Lawrence Berkeley National Laboratory

Jiawei Wan

Lawrence Berkeley National Laboratory

\section{Sophia Betzler}

Lawrence Berkeley National Laboratory

\section{Yujun Xie}

Lawrence Berkeley National Laboratory

\section{Colin Ophus}

Lawrence Berkeley National Laboratory https://orcid.org/0000-0003-2348-8558

\section{Karen Bustillo}

Lawrence Berkeley National Lab https://orcid.org/0000-0002-2096-6078

\section{Peter Ercius}

Lawrence Berkeley National Laboratory https://orcid.org/0000-0002-6762-9976

\section{Lin-wang Wang}

Lawrence Berkeley National Laboratory https://orcid.org/0000-0001-7061-2692

\section{Physical Sciences - Article}

Keywords: Moiré superlattices, bilayer materials, quantum confinement, metavalent bonding, deep energy modulations

Posted Date: February 15th, 2021

DOl: https://doi.org/10.21203/rs.3.rs-211209/v1

License: (9) This work is licensed under a Creative Commons Attribution 4.0 International License. Read Full License 


\title{
Mapping strong electronic coupling in metavalent PbS moiré 2 superlattices
}

3 Yu Wang ${ }^{1,2, \dagger}$, Zhigang Song ${ }^{1, \dagger}$, Jiawei Wan ${ }^{1,2}$, Sophia Betzler ${ }^{1}$, Yujun Xie ${ }^{1}$, Colin Ophus ${ }^{3}$, Karen

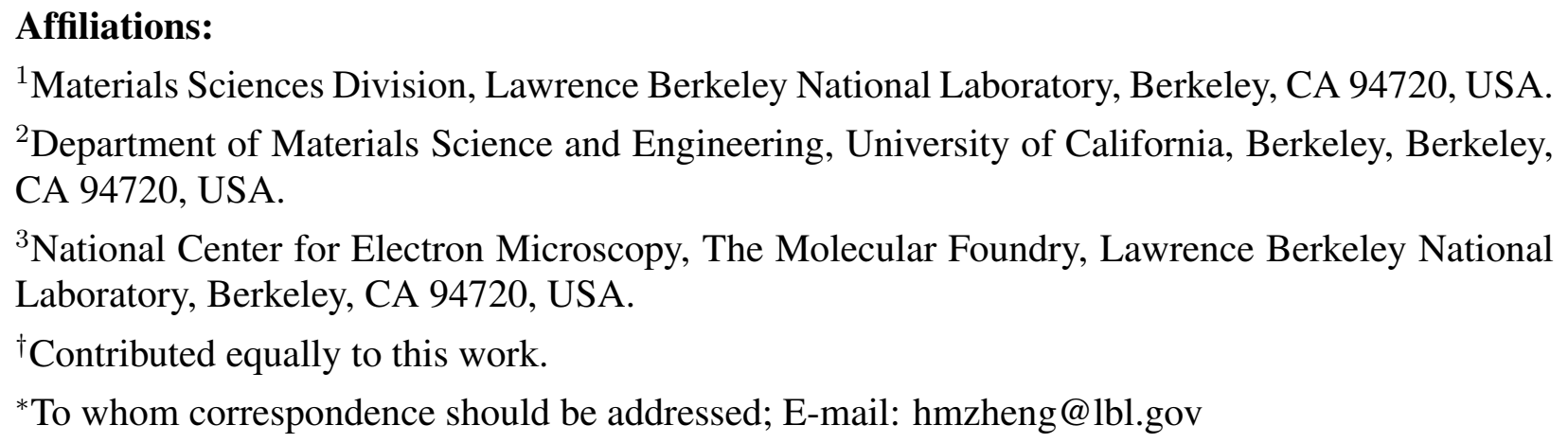

\begin{abstract}
Moiré superlattices are twisted bilayer materials, in which the long-range superlattice potentials from interlayer interactions can create quantum confinement in each layer. The quantum confinement can slow down or localize electrons in moiré superlattices, providing a tunable platform for studying strongly correlated physics ${ }^{1,2}$, such as superconductivity ${ }^{3,4}$, Mott insulators ${ }^{5}$, and interacting topological insulators ${ }^{6,7}$. Previously, moiré superlattices were built exclusively using materials with weak van der Waals interactions ${ }^{8}$ and fabrication of moiré superlattices with strong interlayer chemical bonding (e.g., covalent, metavalent, or ionic bonding) was considered to be impractical ${ }^{9}$. Here using lead sulfide $(\mathrm{PbS})$ as an example, we report a strategy for synthesizing moiré superlattices coupled by strong metavalent bonding. We use water-soluble ligands as a removable template to obtain free-standing ultra-thin $\mathrm{PbS}$ nanosheets and assemble them into direct-contact bilayers with various twist angles. Atomic-resolution imaging shows the interlayer distance is approximately equal to the $\mathrm{Pb}-\mathrm{S}$ bond length, rendering a strong metavalent coupling. Spatial mapping by electron energy loss spectroscopy reveals a strong localization of electronic states in small-angle twisted bilayers, which agrees with our DFT calculations. This study opens a new door to exploration of deep energy modulations within moiré superlattices alternative to van der Waals twistronics.
\end{abstract}




\section{Introduction}

Recently, moiré superlattices have been synthesized by stacking two layers of two-dimensional (2D) materials with relative twist angles ${ }^{9,10}$. In the moiré superlattices, the twisting topology determines the 2D quantum confinement and it offers an additional degree of freedom to modulate the electronic structure, usually referred to as twistronics ${ }^{11,12}$. So far, all $2 \mathrm{D}$ moiré superlattices are synthesized using van der Waals ( $\mathrm{vdW}$ ) materials, such as graphene and transitionmetal dichalcogenide, where the two layers of materials are coupled through vdW interactions. Twistronics based on these vdW materials has attracted great interest in various fields, ranging from physics ${ }^{13-20}$ to materials science ${ }^{9,21-23}$, and chemistry ${ }^{24,25}$. Different from materials coupled by chemical bonding at an interface, such as conventional semiconductor heterostructures, vdW twistronics have reduced strength in modulating the electronic structures due to the weak interlayer coupling. Most of the experimental observations of exotic electronic properties, especially those associated with electron transport, are realized at extremely low temperatures ${ }^{1,2,5-7}$. To increase the electronic modulation imposed by moiré superlattice, one approach is to replace the vdW interactions with strong chemical bonding such as covalent, ionic, or metavalent bonding.

Achieving strong quantum confinement in moiré superlattices by chemical bonding will pave a way to fabricating a new class of materials beyond the current vdW twistronics and it may also shed light on some challenging issues in other systems. For example, strongly coupled moiré superlattices can be structurally and functionally similar to an array of quantum dots ${ }^{26}$, offering an alternative route to super-crystals ${ }^{27}$ by avoiding the notorious issue of connection defects formed during quantum dot self-assembly ${ }^{28}$. Moreover, the energy bands near the Fermi level in moiré patterns can be flattened due to the strong modulation, which may trap electrons in individual "quantum-dot" potentials upon suitable doping, leading to Wigner crystallization ${ }^{29}$. Thus, creating strongly coupled moiré superlattices through chemical bonding combines the strengths of two fields: the tunable confinement of 2D moiré superlattices and the strong coupling in conventional semiconductor heterostructures. Since moiré superlattices cannot be achieved using conventional semiconductor synthesis methods, e.g., epitaxial growth ${ }^{9,30}$, it is unclear whether it is possible to synthesize chemically bonded moiré superlattices.

Here, we use $\mathrm{PbS}$ as a model system to demonstrate a strategy for constructing moiré superlattices with strong interlayer coupling through metavalent bonding. Such chemically bonded moiré superlattices are obtained for the first time. The strong quantum confinement and localization of electronic states in small-angle twisted moiré superlattices are validated through the combination of electron energy loss spectroscopic mapping and theoretical calculations. 


\section{Results and Discussion}

\section{Conceptual discussion of metavalent moiré superlattices}

We first describe theoretically why metavalent moiré superlattices can give rise to stronger coupling effects than vdW moiré superlattices. As shown in Fig. 1a, bulk PbS has a rock-salt crystal structure and features an unconventional metavalent bonding between $\mathrm{Pb}$ and $\mathrm{S}$ atoms, in which the valence electrons are delocalized to an extent between covalent and metallic bonding ${ }^{25}$. This metavalent $\mathrm{Pb}-\mathrm{S}$ bonding is much stronger than $\mathrm{vdW}$ interactions ${ }^{9}$, and it can be used as the interlayer interaction to construct strongly coupled $\mathrm{PbS}$ moiré superlattices if one can assemble ultra-thin $2 \mathrm{D} \mathrm{PbS}$ nanosheets with pristine surfaces into twisted bilayers. This methodology may be further generalized to obtain new moiré superlattices coupled by other types of interfacial chemical bonding, for example, MXene ${ }^{31}$ moiré superlattices coupled by metallic bonding, perovskite ${ }^{32}$ moiré superlattices coupled by ionic bonding, and twisted bilayers of metal-organic frameworks ${ }^{33}$ coupled by mixed interactions.

$\mathrm{PbS}$ moiré supercells with small commensurate angles have a tetragonal symmetry (Fig. 1b), in which two types of interfacial atoms stacked into four general configurations: $\mathrm{Pb}$ on $\mathrm{Pb}$ (marked as $\mathrm{AA}$ ), $\mathrm{Pb}$ on $\mathrm{S}$ (marked as $\mathrm{AB}$ ), the middle point (MP) between $\mathrm{AA}$ and $\mathrm{AB}$, and the diagonal point (DP) between two $\mathrm{AB}$ positions. In small-angle twisted bilayers, each stacking configuration can be approximated by small unit cells consisting of laterally shifted bilayers (Supplementary Information Section 1). Density functional theory (DFT) calculations suggest that different stacking configurations can lead to deep modulations of interlayer distance and bandgap (Fig. 1c). Among these four stacking configurations, AA stacking has the largest interlayer distance, but the smallest bandgap, reflecting distinctive structural and energetic variations stemming from the moiré pattern. Energy modulation by moiré pattern is evaluated by moiré potential ${ }^{17}$, defined as the maximal free energy fluctuation in the real space. We estimate the moire potential by calculating the largest free energy difference among all possible stacking configurations using approximate small unit cells. Fig. 1d shows the calculated moiré potentials of various structures, including the reported vdW superlattices, the metavalent $\mathrm{PbS}$ synthesized in this work, and our predictions of other chemically bonded superlattices. The moiré potential of $\mathrm{PbS}$ is $40 \mathrm{meV}$ per atom, more than twice of the reported vdW superlattices. Generally, chemical bonding leads to much deeper energy modulation compared to vdW interactions. The deep energy modulation can localize electrons in the high-symmetry points with local energy extrema, providing an array of identical quantum-dotlike potentials ${ }^{26,27}$. Until now, the properties of the chemically bonded moire superlattices and their structural stability remains unknown due to the lack of a synthesis strategy. 


\section{Synthesis and characterization of PbS moiré superlattices}

We use metavalent $\mathrm{PbS}$ as a model system of non-vdW moiré superlattices to assess the feasibility of achieving the predicted modulation of electronic structures. Ultra-thin PbS nanosheets have been previously synthesized in organic low-polar solvents ${ }^{34}$, in which the interaction between the solvent-phobic $\mathrm{PbS}$ core and the long-alkyl-chain ligands is designed to be strong to guide asymmetric growth and stabilize the formed nanocrystals. However, the strong core-ligand interaction also leads to difficulty in ligand removal, and the ligands prevent direct metavalent bonding between two nanosheets ${ }^{34}$. To overcome this dilemma, we developed an aqueous synthesis strategy employing two surfactant ligands that have adequate solubility and bind moderately with the inorganic core. The schematic in Fig. 2a shows that $\mathrm{Pb}^{2+}$ and $\mathrm{S}^{2-}$ precursors and two organic ligands (i.e., hexylamine and dodecyl sulfate) in an acidic aqueous solution at $80^{\circ} \mathrm{C}$ for 20 min produce ligand-capped ultra-thin $\mathrm{PbS}$ nanosheets. The synthetic mechanism is discussed in Supplementary Information Section 2. Due to the high polarity of the PbS surfaces, high-polar solvents (such as water) are required to remove the ligands. In this synthesis method, both ligands can be readily removed by washing with dilute basic and acidic aqueous solutions alternatively, in contrast to the long-alkyl-chain ligands used in conventional synthesis. After ligand removal, the naked $\mathrm{PbS}$ nanosheets are immediately drop-casted, allowing the assembly of moiré superlattices through solvent evaporation.

Aberration-corrected transmission electron microscopy (TEM) imaging (Fig. 2b) and the zoomed in portion from the yellow box (Fig. 2c) with corresponding image simulation (Fig. 2c inset) show that the as-synthesized nanosheets have a rock-salt structure with $\{001\}$ surfaces and $\{110\}$ edges. Energy-dispersive X-ray spectroscopy confirms that the moire superlattice consists of $\mathrm{Pb}$ and $\mathrm{S}$ atoms at a molar ratio of 1:1 (Extended Data Fig. 1). Bilayer moiré superlattices with various twist angles are observed at low magnification due to the presence of moiré fringes (Fig. 2d,e). A fast Fourier transform (FFT) of a representative moiré superlattice TEM image shows the expected pattern of two rotated sets of spatial frequencies corresponding to the structure of each sheet (Fig. 2e). In the enlarged images (bottom panels of Fig. 2e), the two red dots correspond to the (200) $d$-spacing of two individual sheets, the set of blue dots correspond to the $d$-spacings of moire pattern, and the orange dots correspond to the addition of the spatial frequencies from individual sheets and the moiré pattern. The theoretical relationship between the FFT pattern of an individual nanosheet and that of a moiré pattern is illustrated in Fig. $2 \mathrm{f}$ inset, and accordingly, the moiré $d$-spacing can be calculated by:

$$
d_{\mathrm{m}}(200)=\frac{d_{\mathrm{rs}}(200)}{2 \cdot \sin (\theta / 2)}
$$

where $d_{\mathrm{m}}$ and $d_{\mathrm{rs}}$ are the (200) $d$-spacing of the moire cell and the rock-salt cell, respectively. In addition, the moiré $d$-spacing can also be directly measured from high-resolution TEM images for 
a variety of twist angles, verifying the calculated results from Eq. 1 as plotted in Fig. 2 f.

Fig. $2 \mathrm{~g}$ and Extended Data Fig. 2 show atomic resolution TEM images and corresponding image simulations (blue images; see Methods) of moiré superlattices with various twist angles. At a large twist angle close to $45^{\circ}$ (measured by rotation of FFT spots), the moiré superlattice resembles 2D octagonal quasicrystals (Extended Data Fig. 2c), showing an approximate $C_{8}$ symmetry in TEM image and reflecting the $S_{8}$ symmetry of octagonal quasicrystals. For smaller twist angles, the (quasi-) unit cell of the moiré pattern is more obvious, and the superlattice appears as an array of identical quantum dots. For example, the moiré superlattice with a $3.2^{\circ}$ twist angle (Fig. $2 \mathrm{~g}$ ) consists of periodic AA/AB regions arranged in square symmetry with DP regions filled in the diagonal positions, resembling an epitaxially fused superlattice of $5 \sim 6 \mathrm{~nm} \mathrm{PbS}$ quantum $\operatorname{dots}^{28}$.

Fig. 2h shows the TEM image along the basal plane (side-view) of a moiré superlattice composed of three PbS nanosheets (labelled as L1, L2, and L3). The three nanosheets have a consistent thickness (approximately $2.7 \mathrm{~nm}$ and consisting of 9 (002) planes). No trace of the ligands is observed between the neighbouring nanosheets, indicating the sheets are in direct contact. Note that, nanosheets L1 and L3 are deformed beyond the right end of L2, indicating strong interlayer metavalent interactions and large deformability of naked $\mathrm{PbS}$ at the sub-10$\mathrm{nm}$ scale $^{35}$. This large deformability of uncoupled nanosheets is consistent with the self-rolling behaviour of individual naked nanosheets in the solution phase (Supplementary Information Section 3). Additionally, this also suggests that forming superlattices is an effective way to stabilize the naked nanosheets.

Further scrutiny of the side-view image in Fig. $2 \mathrm{~h}$ reveals that L1 is oriented along a [110] axis with respect to the viewing direction based on the observed elongated hexagonal pattern, whereas L2 and L3 are tilted away from a low-index zone axis. This indicates that the sheets are rotated around the basal plane with respect to one another, confirming the formation of moiré superlattices with direct interlayer contact. The lattice spacings between each neighbouring (002) plane in the superlattice are measured in real space, based on TEM image simulations and the recognition of the image peak positions, as shown in Fig. 2i and detailed in Supplementary Information Section 4. The (002) spacings are consistent (approximately $3.0 \AA$, similar to that in the bulk crystal) for the inner layers inside each nanosheet, but become slightly larger at the interfaces of nanosheets. The spacing increase at the interface fluctuates at different regions (Extended Data Fig. 3), probably due to the variable interlayer distance for different stacking configurations as suggested by calculations (Fig. 1c and Extended Data Fig. 4). 


\section{Mapping the localization of electronic states}

We employ monochromated electron energy loss spectroscopy (EELS) in an aberration-corrected scanning TEM (STEM) to map the local electronic excitation of moiré superstructures as a function of twist angle. Features in low-loss EEL spectra arise due to inter-band excitation and intraband transitions in a similar way to optical spectra ${ }^{36,37}$. Owing to the advantage of the latest direct detection camera and the large absorption efficiency of $\mathrm{PbS}$, the low-loss spectra in the exciton region exhibit high signal-to-noise ratios (Extended Data Fig. 5). Moreover, using a diskfilter convolution method, we considerably enhance the signal-to-noise ratio for each spectrum corresponding to individual scan positions with a size of 1-2 nm (Extended Data Fig. 5). This allows us to measure local electronic states and optical phenomena of the $\mathrm{PbS}$ nanosheets at the nanoscale.

Fig. 3a,b shows the overview and simultaneously acquired high-angle annular dark field (HAADF-) STEM images, respectively, for a STEM-EELS mapping experiment of a bilayer superlattice with a small twist angle of $1.3^{\circ}$. Analysis of the scattering intensity from nonoverlapping regions with only a single sheet in projection indicates the two nanosheets have a similar thickness with less than $10 \%$ difference. EEL spectra at three representative spots (marked in Fig. 3b) are plotted in Fig. 3c, showing a considerable difference in spectral shape. The spectra at the yellow (single layer), red (double layer), and blue (double layer) spots have their most intense peaks at $1.88,1.79$, and $1.58 \mathrm{eV}$, respectively. The general spectral peak shift to lower energy from single layer to double layer indicates the existence of interlayer electronic coupling and the resulting decrease of bandgaps.

Moreover, the difference in the spectral shape and peak locations between the red and blue positions reflects the variation of band structure within the double layer region. We use a scatter plot (Fig. 3c) and a histogram (Extended Data Fig. 6e) to visualize the distribution of the energies of the most intense peaks for all scanned positions in the single- and double-layer regions. The histogram reveals that the peak energies fall into three bins, i.e., $1.53-1.68 \mathrm{eV}, 1.68-1.83 \mathrm{eV}$, and 1.83-2.0 eV, from which we create a red-blue-yellow colourmap. Using this colourmap, we further map the peak energy spatially in Fig. 3d. The single-layer region gives mostly high-energy peaks over $1.83 \mathrm{eV}$, indicated with yellow in Fig. 3d. The double-layer region shows a segregation of lower-absorption-energy areas (indicated with blue) and higher-absorption-energy areas (indicated with red), reflecting the localization of electronic states in the moiré superlattice. The distance between two neighbouring lower-absorption-energy areas is approximately $25 \mathrm{~nm}$, matching the theoretical (100) moiré $d$-spacing. As shown in Fig. 3d,e, the experimental segregation pattern generally matches the simulated pattern of $\mathrm{AA}, \mathrm{AB}$, and DP configurations of the $1.3^{\circ}$ twisted moiré superlattice (details discussed in Supplementary Information Section 5). This confirms the theoretical prediction in Fig. 1 that strong metavalent interaction can lead to deep energy 
modulation that localizes electrons in the high-symmetry points with local energy extrema.

In experiments on moiré superlattices with larger twist angles (e.g., $3.0^{\circ}$ or $4.6^{\circ}$ shown in Extended Data Fig. 6), STEM-EELS mapping still shows clear shifts to lower energy of spectral peaks from single-layer to double-layer regions. However, the spectral peaks in the double-layer region mostly fall into the bin of $1.68-1.83 \mathrm{eV}$, exhibiting much smaller energy fluctuation than the $1.3^{\circ}$ case. Additionally, no obvious segregation pattern is observed in the mapping of the double-layer region in the cases of $3.0^{\circ}$ or $4.6^{\circ}$ (Extended Data Fig. 6d). The integrated spectra of the double-layer regions with various twist angles are plotted in Fig. 3f, and the peak locations are extracted in Fig. 3g. These plots show that the spectral peak slightly shifts to lower energy as the twist angle decreases from $35.3^{\circ}$ to $3.0^{\circ}$, but the peak energy dramatically decreases from $3.0^{\circ}$ to $1.3^{\circ}$. The spectrum at $1.3^{\circ}$ splits into two peaks because the emergent localization of electronic excitation (Fig. 3d) leads to the separation of spectral peaks in bilayer region (Fig. 3c). By contrast, the spectral peaks in bilayer region are distributed closely together for larger angles (Extended Data Fig. 6c,e). This suggests that the band structure of metavalent moiré superlattices is highly dependent on the twist angle and the electron localization is strongly enhanced at small twist angles.

\section{DFT calculations of PbS moiré superlattices}

We perform systematic DFT calculations to understand the energy modulation of twist angles and the local variations of optical and electronic properties. We evaluated the structural and electronic variations between four specific local configurations ( $\mathrm{AA}, \mathrm{AB}, \mathrm{MP}$, and $\mathrm{DP}$ ) of a moiré superlattice in Fig. 1. Now, we use a similar method to evaluate the transitional configurations between the four extrema by constructing a series of unit cells with continuous lateral shift (Fig. 4a and Supplementary Information Section 1). Fig. 4b-d shows the fluctuations of interlayer distance, free energy, and bandgap as functions of lateral interlayer shift in two dimensions. The surface plot with respect to interlayer distance has the maxima at $\mathrm{AA}$ spots and the minima at $\mathrm{AB}$ spots, and the plot with respect to free energy has a similar shape. The difference between the maximum and minimum interlayer distance is as large as $0.6 \AA$, implying a possible structural reconstruction at the interface in a small-angle twisted superlattice. In contrast to the interlayer distance and energy, the surface plot with respect to the bandgap shows a dramatically different shape with not only more maxima positions but also the minima at AA spots. The bandgap fluctuates up to 0.15 $\mathrm{eV}$, implying a considerable spatial variation of optical absorption or EEL spectra in a small-angle twisted superlattice. The implications of interlayer reconstruction and spatial variation of EEL spectra are in accordance with our experimental observations (Figs. 2i and 3c,d).

We further perform direct self-consistent calculations by constructing large moiré unit cells (up to 4360 atoms) with commensurate angles (Supplementary Information Section 1). The density of 
states (DOS) of the moiré cells with twist angles ranging from $43.6^{\circ}$ to $4.47^{\circ}$ are shown in Fig. $4 \mathrm{e}$, and the corresponding bandgaps are plotted in Fig. 4f. For superlattices with a twist angle larger than $10.4^{\circ}$, the bandgap fluctuates around a constant. However, the bandgap drops rapidly as the twist angle decreases from $10.4^{\circ}$ to smaller angles (Fig. 4f). This calculated relationship between bandgap and twist angle, especially the sharp drop of bandgap at a small angle, is in good agreement with our EELS experiments (Fig. 3g). The reason for the sharp drop of bandgap and the quantitative difference between Fig. $3 \mathrm{~g}$ and Fig. $4 \mathrm{f}$ are revealed by the comparison of the band structures of superlattices with different twist angles and different thickness (Fig. 4h and Extended Data Fig. 7). For smaller twist angles, the bands become narrower due to the quantum containment of the moiré pattern, and bands near the Fermi level separate from the deep and highenergy states. For example, in the case of $3.47^{\circ}$, the bands near the Fermi level, especially the conduction band, become extremely flat (Fig. 4h). In addition, these separated moiré bands at small twist angles are highly spatially localized. Fig. $4 \mathrm{~g}$ shows the corresponding wave functions of the conduction and valence bands, which are localized at $\mathrm{AB}$ and $\mathrm{AA}$ spots, respectively, with diameters of few nanometres. The electronic localization arising from strong interlayer coupling enables our observation of the segregated pattern during STEM-EELS mapping. This also indicates that the PbS moiré superlattice not only structurally resembles but also functionally mimics a wellarranged array of quantum dots with separate energy levels and electron orbitals. Our additional calculation (Supplementary Information Section 6) suggests that the strong metavalent modulation by the moiré pattern may lead to emergent opto-electronic properties in valleytronics, such as valley-dependant optical selection rules.

Furthermore, the structural analysis of the optimized moiré cells (Extended Data Fig. 4ac) confirms the reconstruction at the nanosheet interface. This calculation result suggests that the enlarged interlayer distance at AA spots leads to a fluctuation of interlayer distance in sideviews, matching our experimental observation in Extended Data Fig. 3. In additional calculations (Extended Data Fig. 4a-c), when the thickness of nanosheets increases, the fluctuation of interlayer distance decreases, and the reconstruction mainly occurs near the twisted interface. The thickness increase also leads to the less separated moiré bands (Extended Data Fig. 7), indicating that the modulation effect by moiré pattern becomes weaker as nanosheets become thicker. This explains the quantitative difference between Fig. $3 \mathrm{~g}$ and Fig. 4f, as the structures used in the calculations are thinner than the experimental superlattices for practical reason. The dependence of moiré modulation on the thickness of metavalent nanosheets indicates the value of synthesizing ultrathin free-standing nanosheets, stimulating future developments in the chemical and/or physical syntheses of ultra-thin non-vdW nanosheets and their strongly coupled moiré superlattices. 


\section{Conclusion}

We have established an approach to introduce strong metavalent interlayer interactions into moiré superlattices. It leads to considerable structural reconstruction and electronic renormalization; most interestingly, it gives rise to much stronger electronic localization than that in vdW moiré superlattices. Spatial mapping of the electronic states of $\mathrm{PbS}$ moiré superlattices has been achieved with nanometer resolution using monochromated STEM-EELS. Our synthesis strategy and findings on the chemically bonded moiré superlattices extend the current twistronics. This study provides a route to arrays of identical "quantum-dot" potentials by achieving deep energy modulation through metavalent interactions, providing an alternative platform for spatially variant electronic and opto-electronic properties. We anticipate that further experimental and theoretical studies on non-vdW moiré superlattices will find more types of interlayer interactions that may result in strong electronic coupling, strong correlation, and realizing tunable emergent quantum properties.

\section{Methods}

\section{Synthesis and sample preparation}

$\mathrm{PbS}$ nanosheets were synthesized by mixing a $10 \mathrm{~mL}$ aqueous solution of lead acetate $(20 \mathrm{mM})$, thioacetamide $(20 \mathrm{mM})$, formic acid $(0.3 \mathrm{M})$, sodium dodecyl sulfate $(1 \mathrm{mM})$, and hexylamine $(5 \mathrm{mM})$ at $80^{\circ} \mathrm{C}$ for $20 \mathrm{~min}$. The nanosheet product was centrifuged and washed three times by sodium hydroxide solution $(10 \mathrm{mM}, 1 \mathrm{~mL})$, formic acid solution $(10 \mathrm{mM}, 1 \mathrm{~mL})$, and water $(1 \mathrm{~mL})$ sequentially. The colloid solution of nanosheets was then immediately drop-casted onto holey-carbon TEM grids to form $\mathrm{PbS}$ moiré superlattices. Grids with moiré superlattices were heated at $100^{\circ} \mathrm{C}$ under vacuum for 6 hours before characterization.

\section{TEM imaging and simulation}

The morphology of the moire superlattices was investigated by the FEI ThemIS aberration-corrected TEM at the Molecular Foundry (MF), Lawrence Berkeley National Laboratory (LBNL). Side-view images were obtained through finding the superlattices vertically attached on the edge of holey carbon and further adjusting the view axis by tilting the holder. The interlayer distance was calculated by a custom written MATLAB script, which finds the local maxima of smoothed images and performs subpixel estimation through polynomial interpolation. The TEM images were simulated through a multislice code (compuTEM) with partial coherence (300 keV, $\mathrm{Cs}=0.01 \mathrm{~mm}, 0.5 \mathrm{mrad}$ convergence, $-6 \mathrm{~nm}$ defocus for side-view images and $6 \mathrm{~nm}$ defocus for the rest). 


\section{EELS mapping}

EELS was performed at $300 \mathrm{kV}$ in the TEAM I double-aberration-corrected (scanning) TEM (MF, LBNL) equipped with a high-resolution Continuum Gatan Imaging Filter (GIF) spectrometer and a $4 \mathrm{k} \times 4 \mathrm{k}$ Gatan K3 direct electron detector. For survey imaging, the HAADF detector was used in the STEM system. The relative thickness of nanosheets was estimated by comparing the local image intensity, and the twisted angles are measured by the edge of nanosheets in real space. EELS maps were acquired in the same STEM mode by using an electron beam probe $0.1 \mathrm{~nm}$ in diameter with $150 \mathrm{meV}$ energy resolution. The step size used in $1.3^{\circ}, 3.0^{\circ}$, and $4.6^{\circ}$ cases was 2 , 4 , and 1 $\mathrm{nm}$, respectively. The energy dispersion was set to $9 \mathrm{meV}$ per channel and the dual EELS mode was used to eliminate any systematic error due to zero-loss shift.

The EELS dataset was analysed using custom written scripts in MATLAB. For showing the spectral variation in each mapping, a disk filter method was developed for enhancing the signal-tonoise ratio of local spectra. For comparing the spectral difference for different moiré superlattices, Savitzky-Golay smoothing, Richardson-Lucy deconvolution, and zero-loss-peak removal were applied. Each processed spectrum was manually checked to preserve the feature of the original spectrum. Data processing details are shown in Extended Data Fig. 5.

\section{Theoretical calculations}

All calculations in this work are self-consistent calculations. The calculations of the variations of energy, interlayer distance, and bandgap upon the interlayer lateral shift were performed using plane-wave code package PWmat, and SG15 was used. The exchange and correlation functional is described by the Perdew-Burke-Ernzerhof (PBE) generalized gradient approximation (GGA). Because the band edges are not at high-symmetric points, we calculated the bandgap based on selfconsistent calculations using a dense k-mesh rather than reading the bandgap from band structure. The k-point mesh was sampled by the Monkhorst-Pack method with a separation smaller than 0.01 $\AA^{-1}$

Because most commensurate moiré supercells contain more than 1000 atoms (4360 in the largest calculated supercell), we used the atomic basis set of single-Zeta functions and FHI pseudopotentials in the direct calculations. This atomic basis set was tested to be accurate by comparing with the plane-wave method in small systems.

In all calculations, a vacuum layer thicker than $20 \AA$ was applied to avoid the periodic images. All geometry and ions were fully relaxed until the force of each atom decreases to $0.01 \mathrm{eV} / \AA$. To include the possible vdW interaction between different layers, the DFT-D2 functional was implemented. 


\section{Data availability}

The data that support the findings of this study are available from the corresponding authors on reasonable request.

\section{References}

[1] Tang, Y. et al. Simulation of Hubbard model physics in $\mathrm{WSe}_{2} / \mathrm{WS}_{2}$ moiré superlattices. Nature 579, 353-358 (2020).

[2] Lu, X. et al. Superconductors, orbital magnets and correlated states in magic-angle bilayer graphene. Nature 574, 653-657 (2019).

[3] Cao, Y. et al. Unconventional superconductivity in magic-angle graphene superlattices. Nature 556, 43-50 (2018).

[4] Arora, H. S. et al. Superconductivity in metallic twisted bilayer graphene stabilized by WSe . Nature 583, 379-384 (2020).

[5] Cao, Y. et al. Correlated insulator behaviour at half-filling in magic-angle graphene superlattices. Nature 556, 80-84 (2018).

[6] Nuckolls, K. P. et al. Strongly correlated Chern insulators in magic-angle twisted bilayer graphene. Nature 588, 610-615 (2020).

[7] Chen, G. et al. Tunable correlated Chern insulator and ferromagnetism in a moiré superlattice. Nature 579, 56-61 (2020).

[8] Carr, S., Fang, S. \& Kaxiras, E. Electronic-structure methods for twisted moiré layers. Nat. Rev. Mater. 5, 748-763 (2020).

[9] Liu, Y., Huang, Y. \& Duan, X. Van der Waals integration before and beyond two-dimensional materials. Nature 567, 323-333 (2019).

[10] Bediako, D. K. et al. Heterointerface effects in the electrointercalation of van der Waals heterostructures. Nature 558, 425-429 (2018).

[11] Ribeiro-Palau, R. et al. Twistable electronics with dynamically rotatable heterostructures. Science 361, 690-693 (2018).

[12] $\mathrm{Hu}, \mathrm{G}$. et al. Topological polaritons and photonic magic angles in twisted $\alpha-\mathrm{MoO}_{3}$ bilayers. Nature 582, 209-213 (2020). 
[13] Dean, C. R. et al. Hofstadter's butterfly and the fractal quantum hall effect in moiré superlattices. Nature 497, 598-602 (2013).

[14] Jiang, Y. et al. Charge order and broken rotational symmetry in magic-angle twisted bilayer graphene. Nature 573, 91-95 (2019).

[15] Uri, A. et al. Mapping the twist-angle disorder and landau levels in magic-angle graphene. Nature 581, 47-52 (2020).

[16] Sunku, S. S. et al. Photonic crystals for nano-light in moiré graphene superlattices. Science 362, 1153-1156 (2018).

[17] Tran, K. et al. Evidence for moiré excitons in van der Waals heterostructures. Nature 567, 71-75 (2019).

[18] Alexeev, E. M. et al. Resonantly hybridized excitons in moiré superlattices in van der Waals heterostructures. Nature 567, 81-86 (2019).

[19] Seyler, K. L. et al. Signatures of moiré-trapped valley excitons in $\mathrm{MoSe}_{2} / \mathrm{WSe}_{2}$ heterobilayers. Nature 567, 66-70 (2019).

[20] Sharpe, A. L. et al. Emergent ferromagnetism near three-quarters filling in twisted bilayer graphene. Science 365, 605-608 (2019).

[21] Sutter, P., Wimer, S. \& Sutter, E. Chiral twisted van der Waals nanowires. Nature 570, 354-357 (2019).

[22] Rhodes, D., Chae, S. H., Ribeiro-Palau, R. \& Hone, J. Disorder in van der Waals heterostructures of 2D materials. Nat. Mat. 18, 541-549 (2019).

[23] Weston, A. et al. Atomic reconstruction in twisted bilayers of transition metal dichalcogenides. Nat. Nanotech. 15, 592-597 (2020).

[24] Dong, R., Zhang, T. \& Feng, X. Interface-assisted synthesis of 2D materials: Trend and challenges. Chem. Rev. 118, 6189-6235 (2018).

[25] Kooi, B. J. \& Wuttig, M. Chalcogenides by design: Functionality through metavalent bonding and confinement. Adv. Mater. 32, 1908302 (2020).

[26] Song, Z., Sun, X. \& Wang, L. Switchable asymmetric moiré patterns with strongly localized states. J. Phys. Chem. Lett. 11, 9224-9229 (2020).

[27] Zhang, C. et al. Interlayer couplings, Moiré patterns, and 2D electronic superlattices in $\mathrm{MoS}_{2} / \mathrm{WSe}_{2}$ hetero-bilayers. Sci. Adv. 3, e1601459 (2017). 
[28] Whitham, K. et al. Charge transport and localization in atomically coherent quantum dot solids. Nat. Mat. 15, 557-563 (2016).

[29] Wigner, E. Effects of the electron interaction on the energy levels of electrons in metals. Trans. Faraday Soc. 34, 678-685 (1938).

[30] Oh, M. H. et al. Design and synthesis of multigrain nanocrystals via geometric misfit strain. Nature 577, 359-363 (2020).

[31] Naguib, M., Mochalin, V. N., Barsoum, M. W. \& Gogotsi, Y. 25th anniversary article: MXenes: a new family of two-dimensional materials. Adv. Mater. 26, 992-1005 (2014).

[32] Ji, D. et al. Freestanding crystalline oxide perovskites down to the monolayer limit. Nature 570, 87-90 (2019).

[33] Wu, M. et al. Conetronics in 2D metal-organic frameworks: double/half Dirac cones and quantum anomalous Hall effect. 2D Mater. 4, 015015 (2016).

[34] Schliehe, C. et al. Ultrathin PbS sheets by two-dimensional oriented attachment. Science 329, 550-553 (2010).

[35] Wang, Y. et al. Dynamic deformability of individual PbSe nanocrystals during superlattice phase transitions. Sci. Adv. 5, eaaw5623 (2019).

[36] Hage, F. S. et al. Nanoscale momentum-resolved vibrational spectroscopy. Sci. Adv. 4, eaar7495 (2018).

[37] Gogoi, P. K. et al. Layer rotation-angle-dependent excitonic absorption in van der Waals heterostructures revealed by electron energy loss spectroscopy. ACS Nano 13, 9541-9550 (2019).

\section{Acknowledgements}

The work was supported by the U.S. Department of Energy (DOE), Office of Science, Office of Basic Energy Sciences (BES), Materials Sciences and Engineering Division under Contract No. DE-AC02-05-CH11231 within the $\mathrm{KC} 22 \mathrm{ZH}$ program. Y.W. was partially supported by the UC Office of the President under the UC Laboratory Fees Research Program Collaborative Research and Training Award LFR-17-477148. Work at the Molecular Foundry was supported by the Office of Science, Office of Basic Energy Sciences, of the U.S. Department of Energy under Contract No. DE-AC02-05CH11231. 


\section{Author contributions}

Y.W. conceived and H.Z. supervised this project. Y.W. and J.W. designed and performed the synthesis. Y.W. performed TEM imaging with contribution from Y.X. P.E. and Y.W. conducted EELS mapping with contribution from S.B. and K.B. C.O. provided the code for analysing sideview images. Y.W. analysed all experimental data. Z.S. performed theoretical analysis under the supervision of L.-W.W. Y.W., Z.S., and H.Z. wrote the manuscript with input from all authors.

\section{Competing interests}

The authors declare no competing interests.

\section{Materials \& Correspondence}

Supplementary information is available for this paper at https://doi.org/. Correspondence and requests for materials should be addressed to Name Surname. 


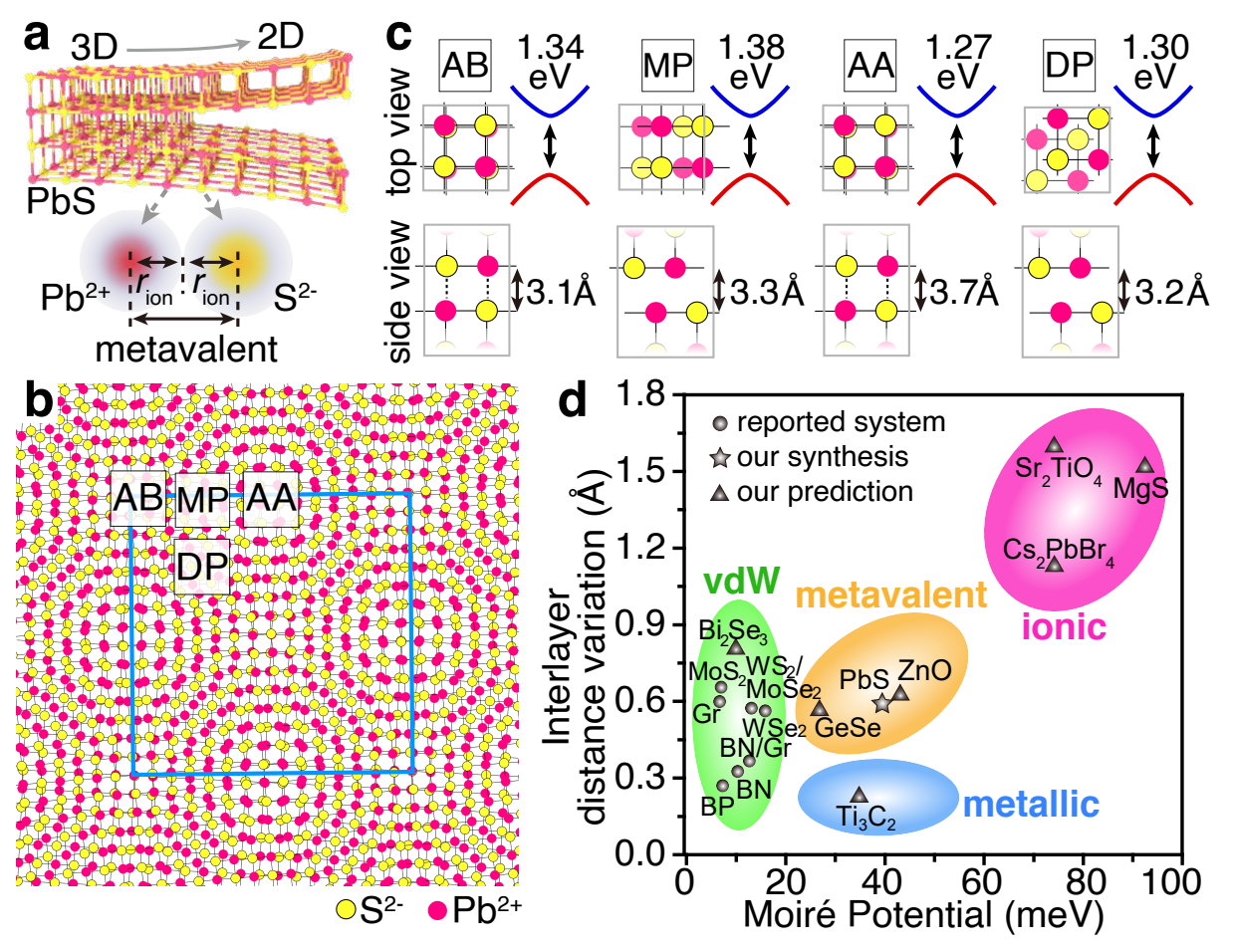

Fig. 1 Structure and strong coupling of PbS moiré superlattice. a, 3D and the cleaved 2D structures of $\mathrm{PbS}$ rock-salt crystal, emphasizing the metavalent interaction in all directions. b, Different local atomic alignments occur in a $\mathrm{PbS}$ moiré superlattice with a twist angle of $8^{\circ}$. Blue square marks the moiré unit-cell. Four representative stacking configurations are highlighted as $\mathrm{Pb}$ on $\mathrm{Pb}$ (AA), $\mathrm{Pb}$ on $\mathrm{S}(\mathrm{AB})$, middle point (MP), and diagonal point (DP). c, DFT calculations of AA, $\mathrm{AB}, \mathrm{MP}$, and DP configurations, showing the structure, bandgap, and interfacial distance of each configuration. d, DFT calculations on varieties of moiré superlattices, including the reported vdW superlattices, the metavalent $\mathrm{PbS}$ synthesized in this work, and our predictions of other chemically bonded superlattices. The results show their moiré potential and the largest interfacial distance variation among different stacking configurations. 

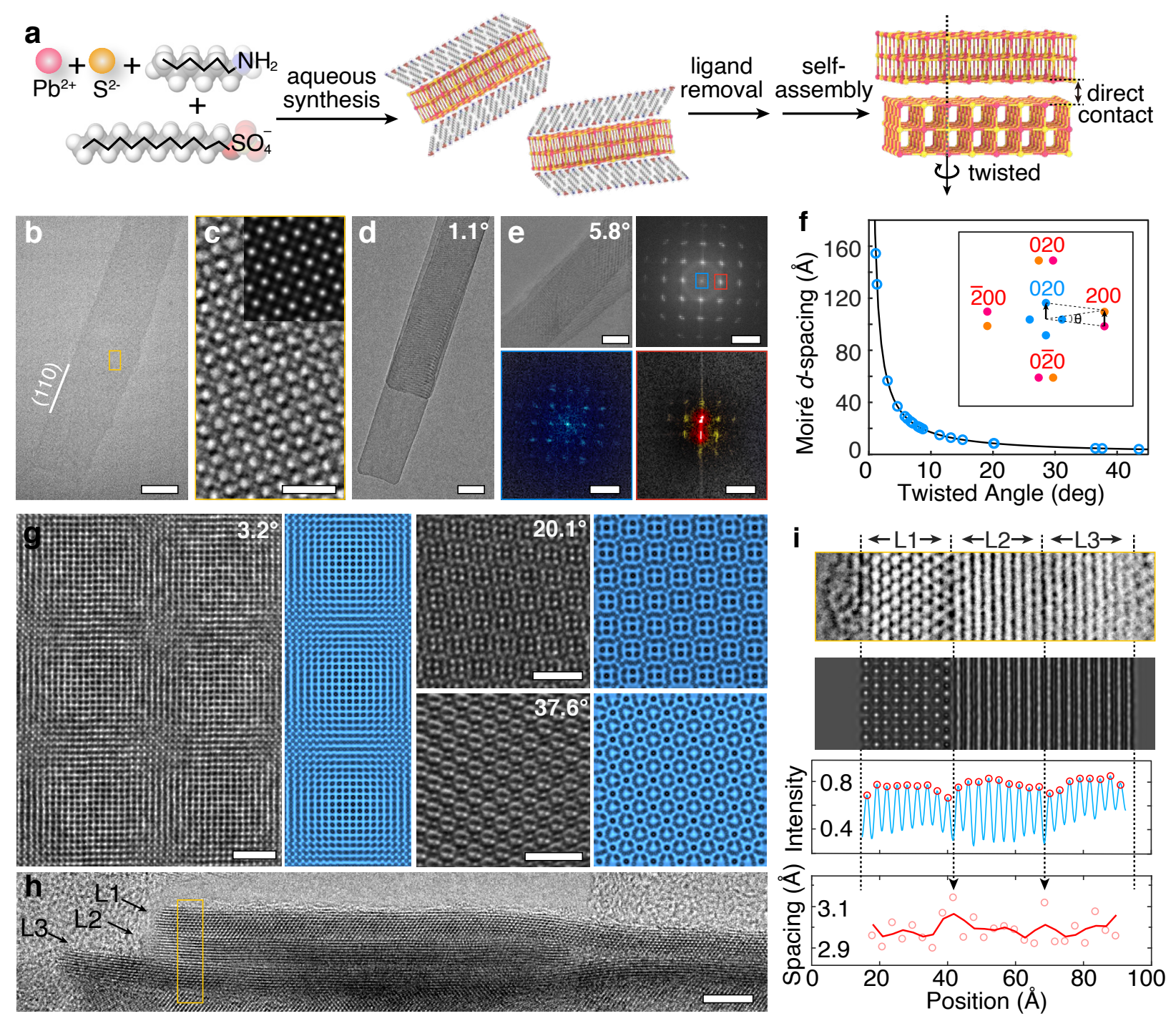

Fig. 2 Synthesis and TEM characterizations of PbS moiré superlattices. a, Schematics of synthetic procedures. b, Low magnification TEM image of a single ultra-thin $\mathrm{PbS}$ nanosheet. c, Atomic resolution image of the boxed area in panel $b$ with an inserted simulation of TEM image. d, TEM image of a bilayer moiré superlattice with a $1.1^{\circ}$ twist angle. e, TEM image (top-left) and FFT pattern (top-right) of a bilayer moiré superlattice with a $5.8^{\circ}$ twist angle. Two bottom panels show the enlarged details of the FFT pattern, in which blue dots indicate moiré spatial frequencies, red dots indicate the (200) spatial frequencies of two individual rock-salt nanosheets, and orange dots indicate the emerged pattern from moiré pattern and individual rock-salt patterns. f, Theoretical (black curve) and observed (green hollow dots) relationship between moiré $d$-spacing (200) and twist angles. Inset shows the relationship between moiré spatial frequencies (green) and two sets of individual rock-salt spatial frequencies (red and orange). g, Atomic resolution TEM images and corresponding simulated images (false coloured) of bilayer moiré superlattices with a variety of twist angles. $\mathbf{h}$, Side view of a moiré superlattice composed of three layers, L1, L2, and L3. The region marked by the orange box is flipped and zoomed in panel i. i, Structural analysis of side-view details. From top to bottom are original TEM image, simulated image, image intensity and peak position of each atomic layer, and each interlayer spacing. Scale bar: b, $30 \mathrm{~nm}$; c, $1 \mathrm{~nm}$; $\mathrm{d}, 100 \mathrm{~nm}$; e, $30 \mathrm{~nm}$ (top left), $5 \mathrm{~nm}^{-1}$ (top right), $0.5 \mathrm{~nm}^{-1}$ (bottom two); g, $2 \mathrm{~nm}$; h, $5 \mathrm{~nm}$. 

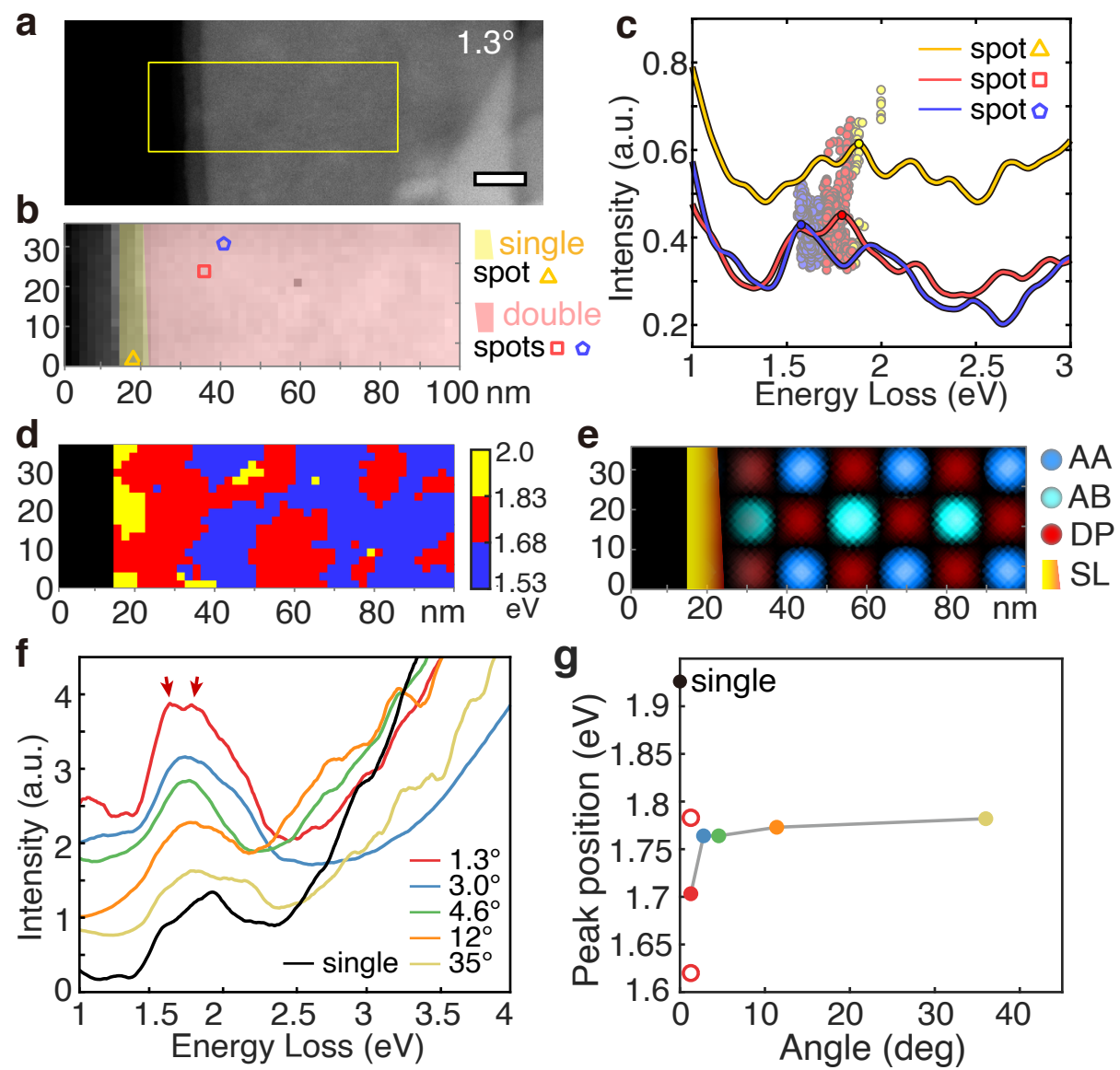

Fig. 3 Mapping of electronic states through STEM-EELS. a, Overview image of a moiré superlattice with a twist angle of $1.3^{\circ}$. Yellow box marks the scanning region for STEM-EELS. Scale bar, $20 \mathrm{~nm}$. b, Scanned image with single- and double-layer regions false-coloured and three representative spots marked. c, Plot of low-loss spectra from three representative spots with the spectral peaks marked with opaque dots. For other scanned spots, the spectra are omitted for clarity, only their spectral peaks are illustrated by the semi-transparent dots. Dot colour follows the colourmap in d. d, Spatial mapping of the energy of spectral peaks that are shown by scattered dots in c. e, Simulated pattern of AA, AB, and DP configurations and single layer (SL) region in the moiré superlattice with a twist angle of $1.3^{\circ}$. f, Integrated spectra of the double-layer regions of moiré superlattices with a variety of twist angles. A spectrum of single-layer $\mathrm{PbS}$ nanosheet is added for comparison. g, The change of the peak energy of double-layer regions upon the change of the twist angle. Hollow dots at $1.3^{\circ}$ shows the position of two separated peaks (marked by red arrows in panel f), whereas the solid dot shows the average of the two peaks. Energy dispersion of all EEL spectra is $9 \mathrm{meV} /$ pixel. 

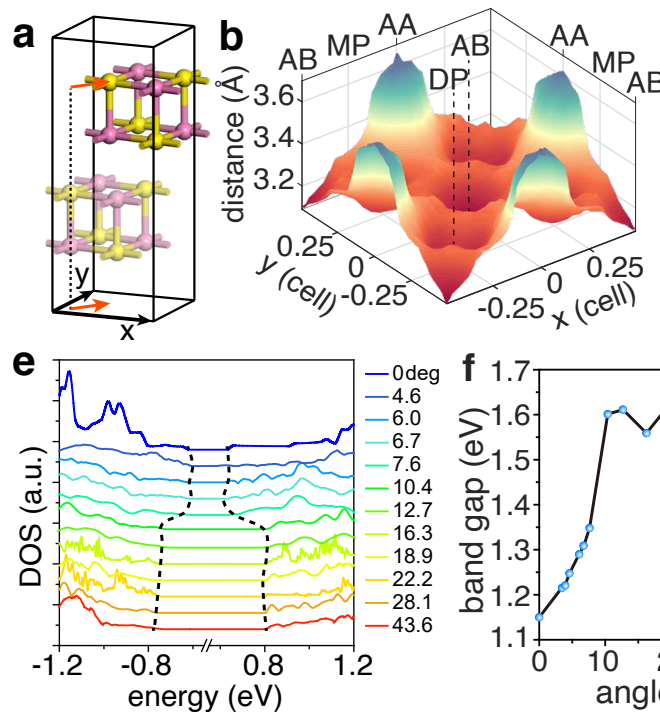

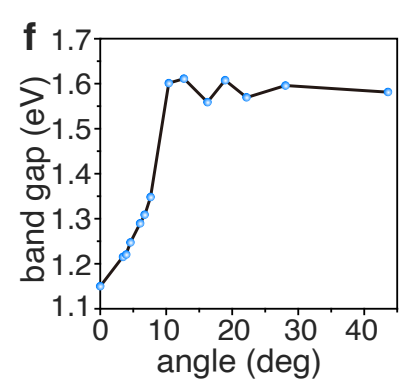

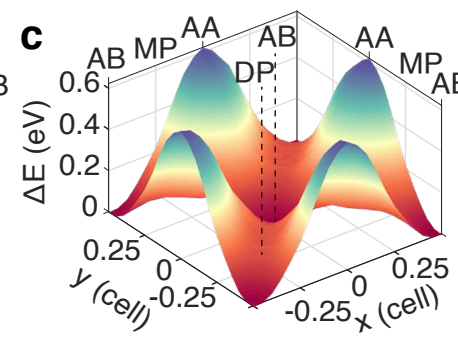

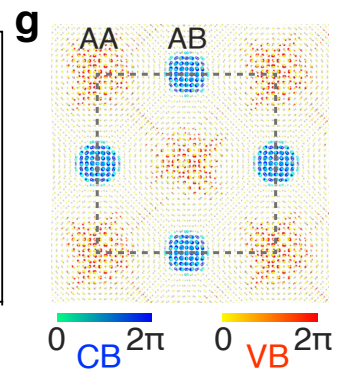

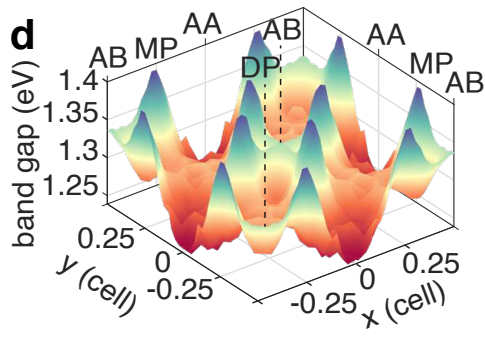

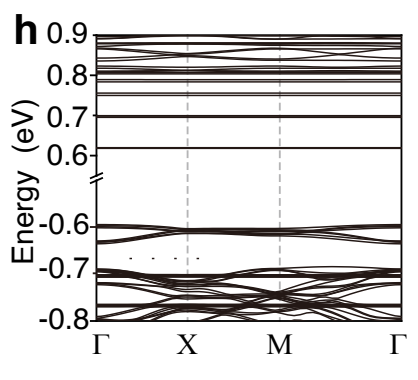

Fig. 4 DFT calculations of the electronic localization. a, Illustration of shifting two $\mathrm{PbS}$ nanosheets for creating different stacking configurations and calculating its impact on the structural and electronic properties. b-d, Change of the interfacial distance (b), free energy (c), and bandgap (d) upon the bilayer lateral shift. $x$ and $y$ are the fractional coordinates of the lateral shift. e, Direct calculation of the density-of-states of commensurate moiré superlattices with a variety of twist angles. Black dashed lines indicate the positions of first valence and conduction bands. $\mathbf{f}$, Bandgap change upon twist angles extracted from panel e. g, Charge density of valence band (CB) and conduction band (VB). Colour is coded by the phase of wave function. $\mathbf{h}$, Band structure of the moiré superlattice with a $3.47^{\circ}$ twist angle. 


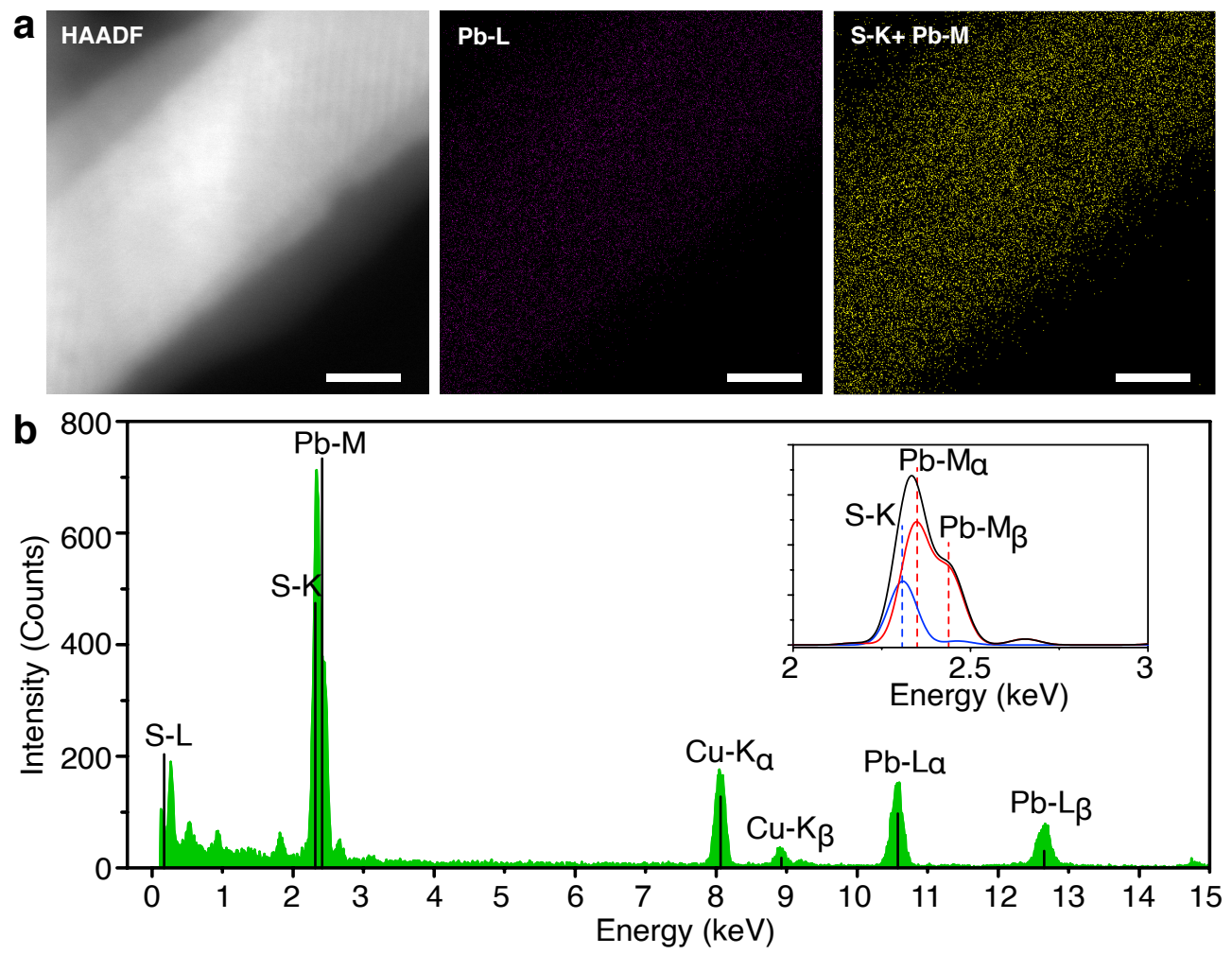

Extended Data Fig. 1 EDS of PbS moiré superlattice. a, HAADF STEM image and Pb and S element mapping images of a moiré superlattice with a twist angle of $6.5^{\circ}$. All scale bars are 20 $\mathrm{nm}$. The uniform contrast in the STEM and element mapping images suggests no segregation of $\mathrm{Pb}$ and $\mathrm{S}$. b, Energy dispersive spectrum of the moiré superlattice. Inset shows the deconvolution of $\mathrm{S}-\mathrm{K}$ and $\mathrm{Pb}-\mathrm{M}$ peaks. Quantification of the atomic fractions reveals $48 \% \mathrm{~Pb}$ (using the L peaks) and $52 \% \mathrm{~S}$ (using the deconvoluted $\mathrm{K}$ peak). 

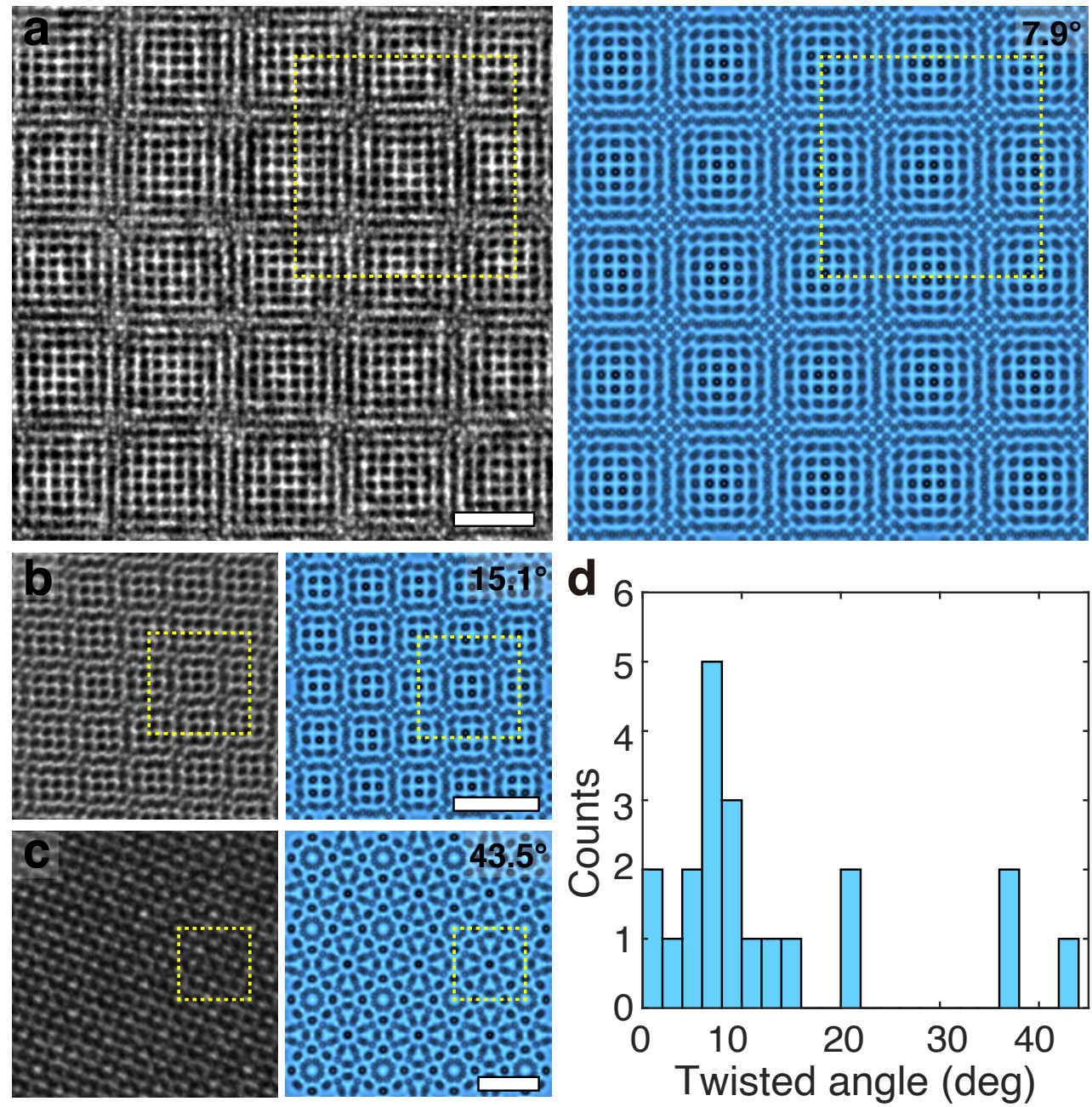

Extended Data Fig. 2 Atomic-resolution imaging and the statistics of moiré patterns. ac, Representative TEM images (left) and corresponding simulated images (right, false coloured) of $\mathrm{PbS}$ moiré superlattices with various twist angles. Yellow boxes mark the quasi-unit cells. d, Statistics of the twist angles of 21 moiré superlattices based on atomic resolution imaging. Scale bars: a,b, $2 \mathrm{~nm}$; c, $1 \mathrm{~nm}$. 

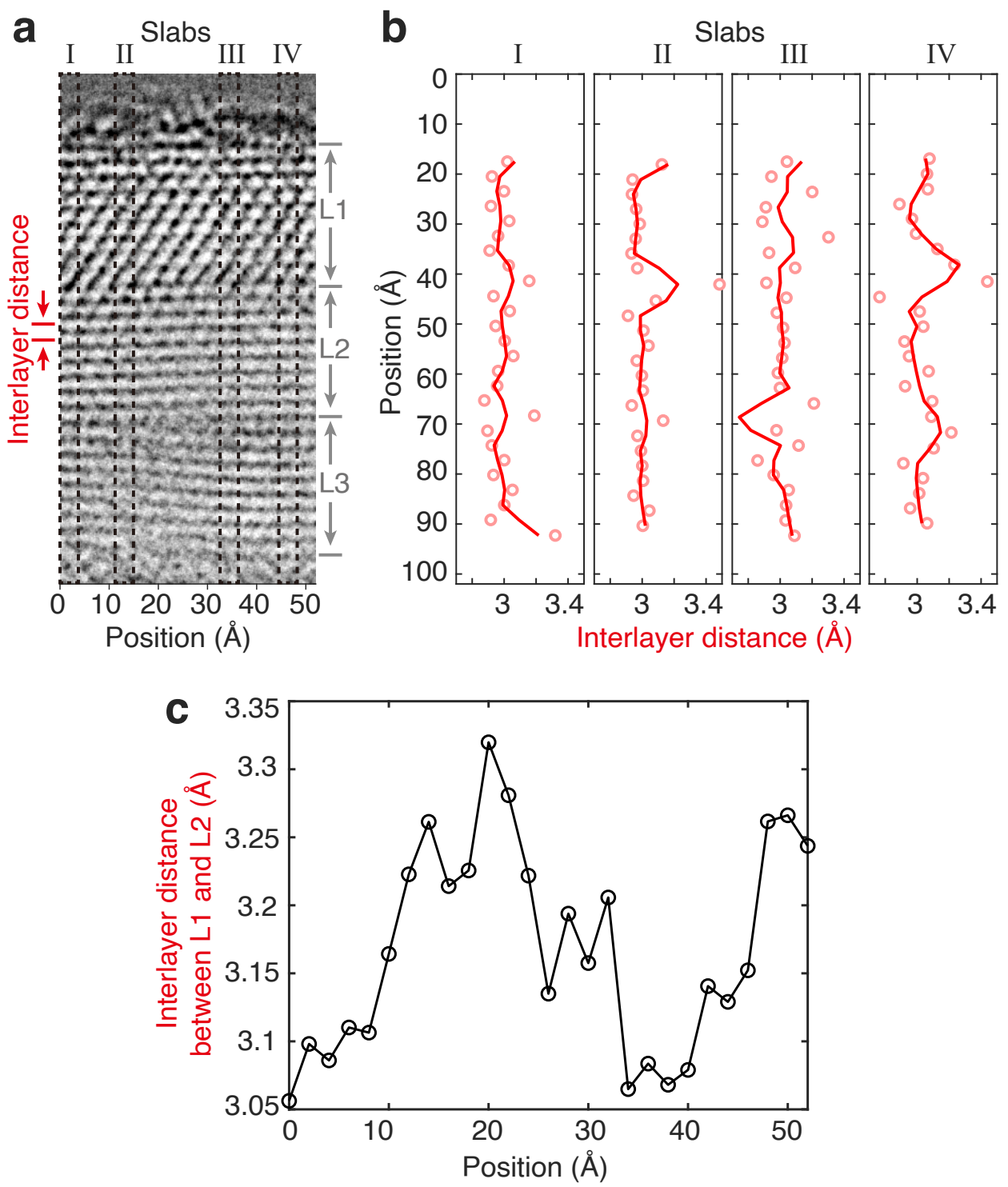

Extended Data Fig. 3 Fluctuation of the interlayer distance of moiré superlattice revealed by side-view-image analysis. a, False-coloured side-view TEM image of a part of the PbS moiré superlattice shown in Fig. 2h. The boundaries of three nanosheets are indicated by green arrows, and one representative interlayer distance is indicated by red arrows. Local interlayer distances at each position ( 0 to $50 \AA)$ are evaluated by analysing the image intensity in corresponding slabs with a $4 \AA$ width. Four representative slabs at the positions of $2,12,34$, and $46 \AA$ are boxed and labelled for illustration. $\mathbf{b}$. Interlayer distance in each slab extracted from the image intensity analysis through a similar method shown in Fig. 2i. c, Fluctuation of the interlayer distance at the interface between L1 and L2 at different positions. 

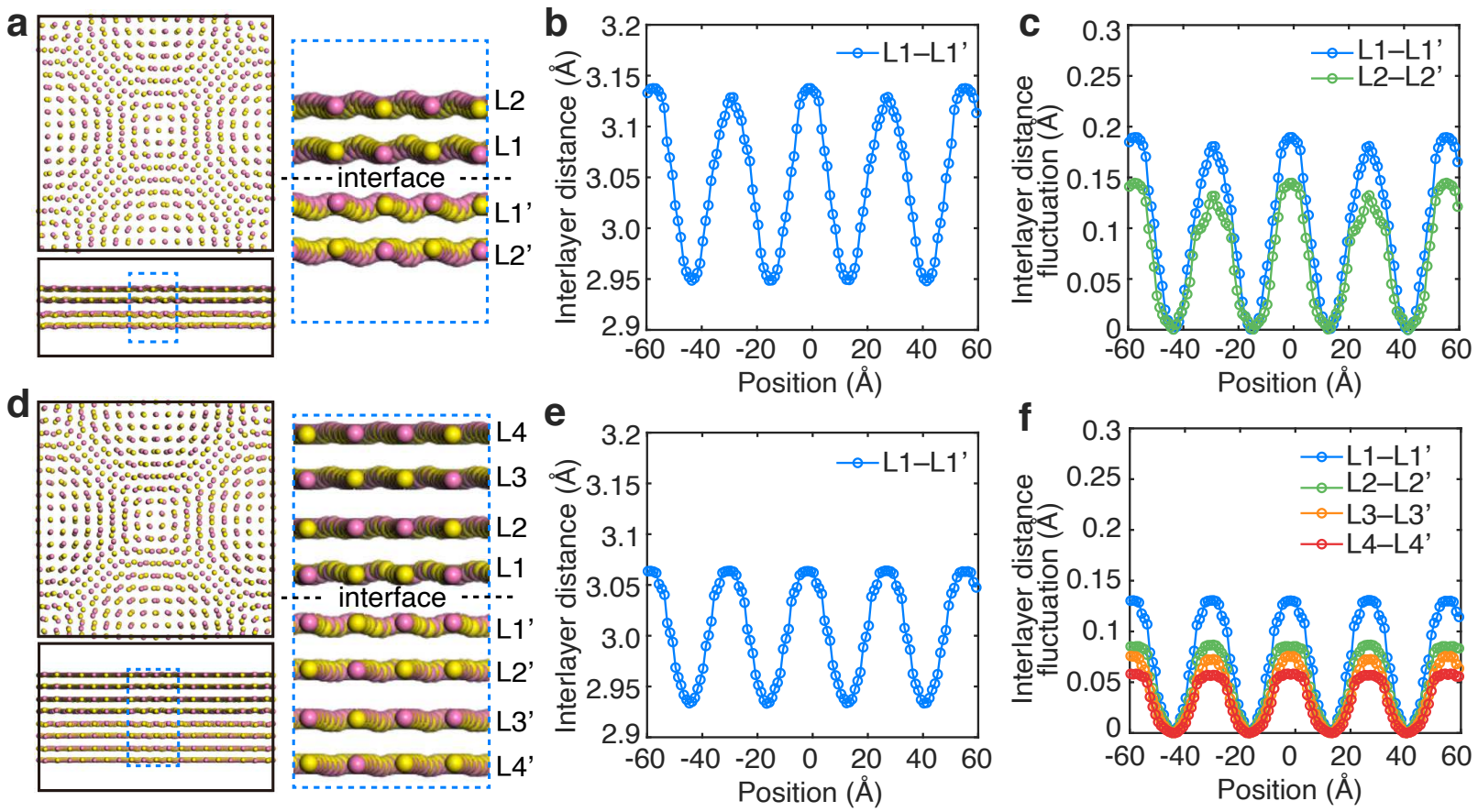

Extended Data Fig. $4 \mid$ Structural reconstruction of moiré superlattice. Panels a-c and $\mathbf{d}-\mathbf{f}$ correspond to the calculation results of $6.06^{\circ}$ twisted moiré cells consisting of bilayer 2-atomthick nanosheets $(\mathrm{a}-\mathrm{c})$ or bilayer 4-atom-thick nanosheets (d-f). a,d, Top view, side view, and enlarged side view of the relaxed structures. Interfaces are marked by black dash lines and each atom layer is sequentially labelled from the interfaces to the vacuum slabs. b,e, Interfacial distance between L1- L1' measured from the side view. c,f, Fluctuations of the interlayer distance between counter layers, i.e., L1-L1' to L4-L4', measured from the side view. 

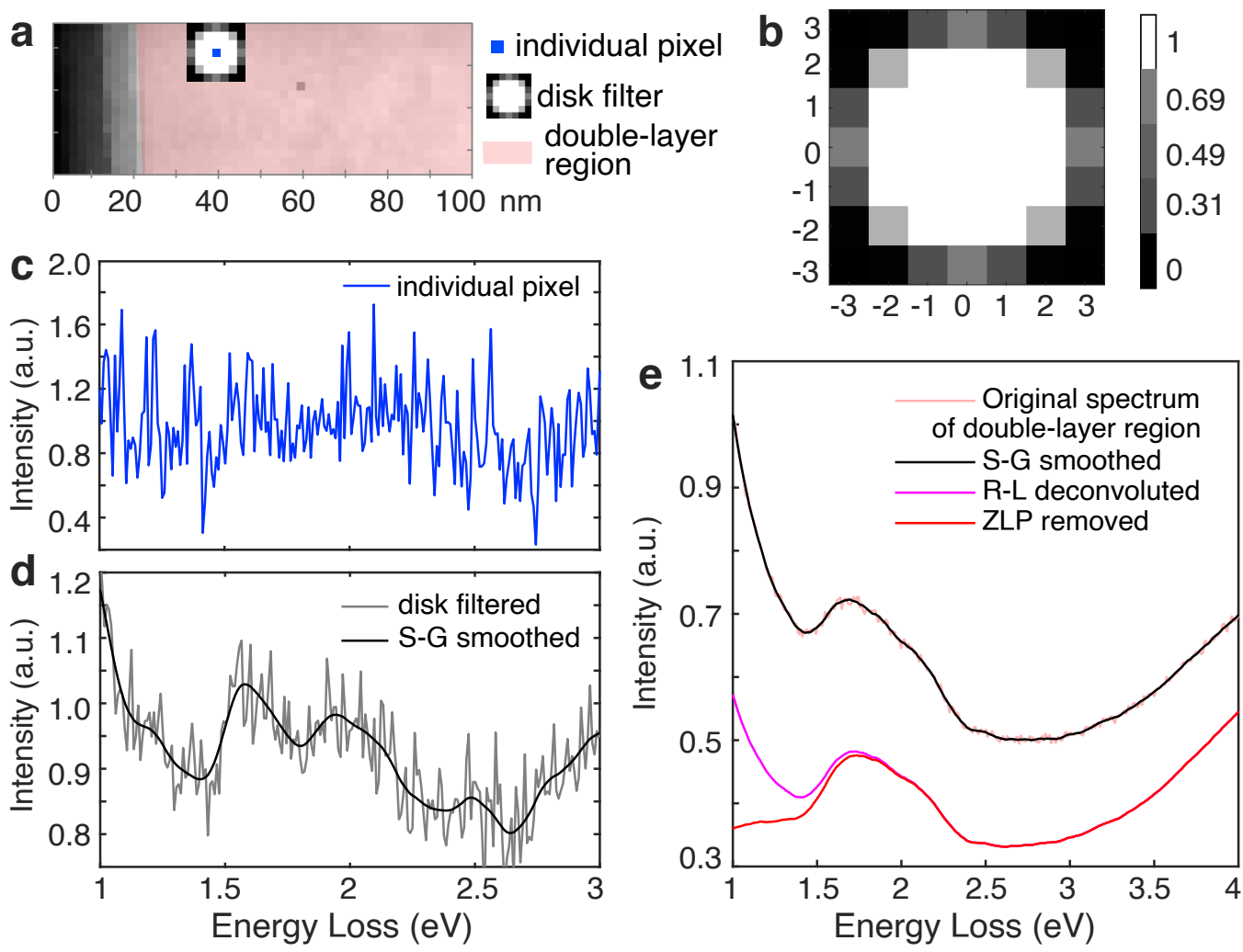

Extended Data Fig. 5 Data processing method for EELS mapping. a, Illustration of the areas for extracting an individual spectrum, the corresponding disk-filtered spectrum, and the normalized spectrum of the overall double-layer region. b, Detail of the $7 \times 7$ (pixel) disk kernel for filtering. c, Noisy spectrum extracted from the original data at the pixel labelled in panel a. d, Disk-filtered and further smoothed spectra corresponding to the same pixel, but with a much higher signal-tonoise ratio. Filtering is implemented by convolution of original 3D data with the disk kernel shown in panel b, and smoothing is based on Savitzky-Golay method. e, Processing of the normalized spectrum of the overall double-layer region. The integration of the original spectra shows an extremely high signal-to-noise ratio, therefore, the further processing methods (such as SavitzkyGolay smoothing, Richardson-Lucy deconvolution, and zero-loss-peak removal through powerlaw fitting) preserve the feature of the original spectrum. Energy dispersion of all spectra in panels c-e is $9 \mathrm{meV} / \mathrm{pixel}$. 

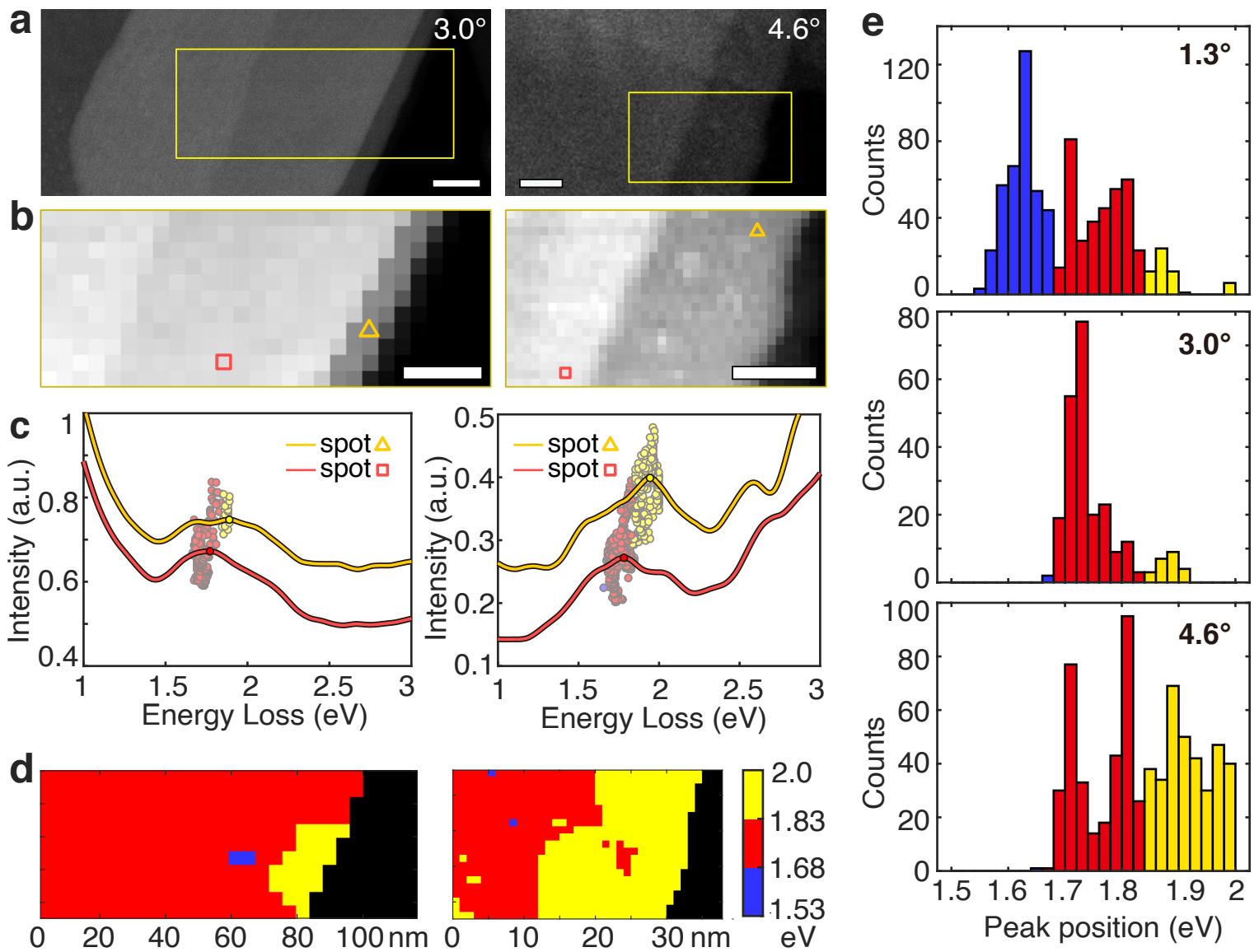

Extended Data Fig. 6 EELS mapping of moiré superlattices. Left and right columns of ad correspond to the moiré superlattices with twisted angles of $3.0^{\circ}$ and $4.6^{\circ}$, respectively. All nanosheets measured in EELS mapping have a similar thickness with less than $10 \%$ difference based on the analysis of the scattering intensity in overview images. Scale bars are $20 \mathrm{~nm}$ (left column) and $10 \mathrm{~nm}$ (right column). a, Survey image of the moiré superlattices. Yellow box marks the scanning region for STEM-EELS. b, Scanned images with two representative spots in singleand double-layer regions, marked with triangle and square, respectively. c, Plot of low-loss spectra from two representative spots. For other scanned spots, the spectra are omitted for clarity, only their spectral peaks between 1.3 to $2.5 \mathrm{eV}$ are illustrated by the scattered dots. Dot colour follows the colourmap in d. d, 2D mapping of the energy of spectral peaks of all scanned pixels. Colourmap is identical to that in Fig. 3d. e, Histogram of the peak positions of the absorption spectra in the EELS measurements of three moire superlattices with different twist angles (labelled). Histograms correspond to the scatter plots in Fig. 3c and Extended Data Fig. 6c. 


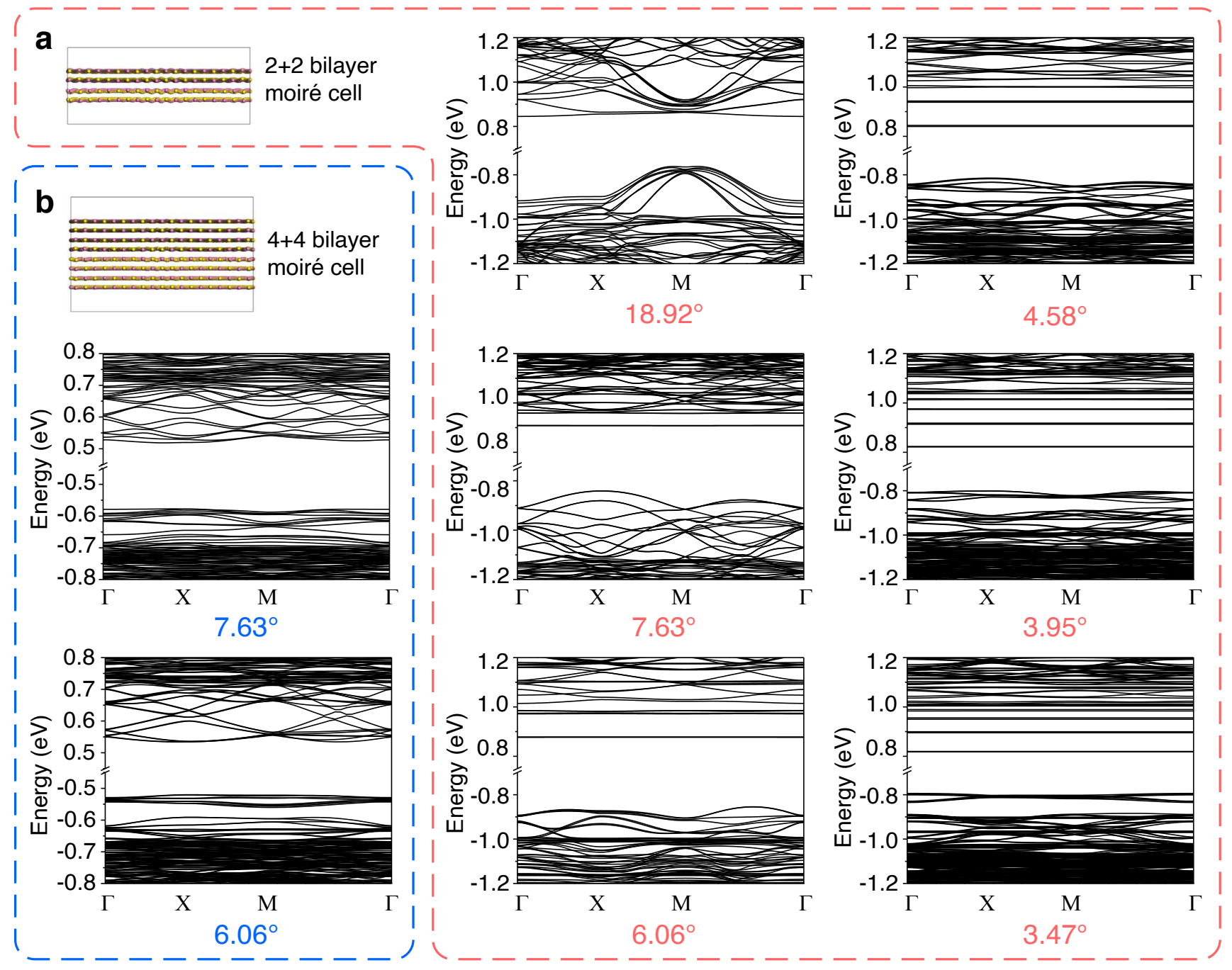

Extended Data Fig. 7 Band structures directly calculated from moiré cells with commensurate angles. Energy dispersion along the high symmetric path for moiré cells constructed from bilayer 2-atom-thick nanosheets (a) or bilayer 4-atom-thick nanosheets (b). A variety of commensurate angles are calculated for showing the influence of twist angles and nanosheet thickness. 

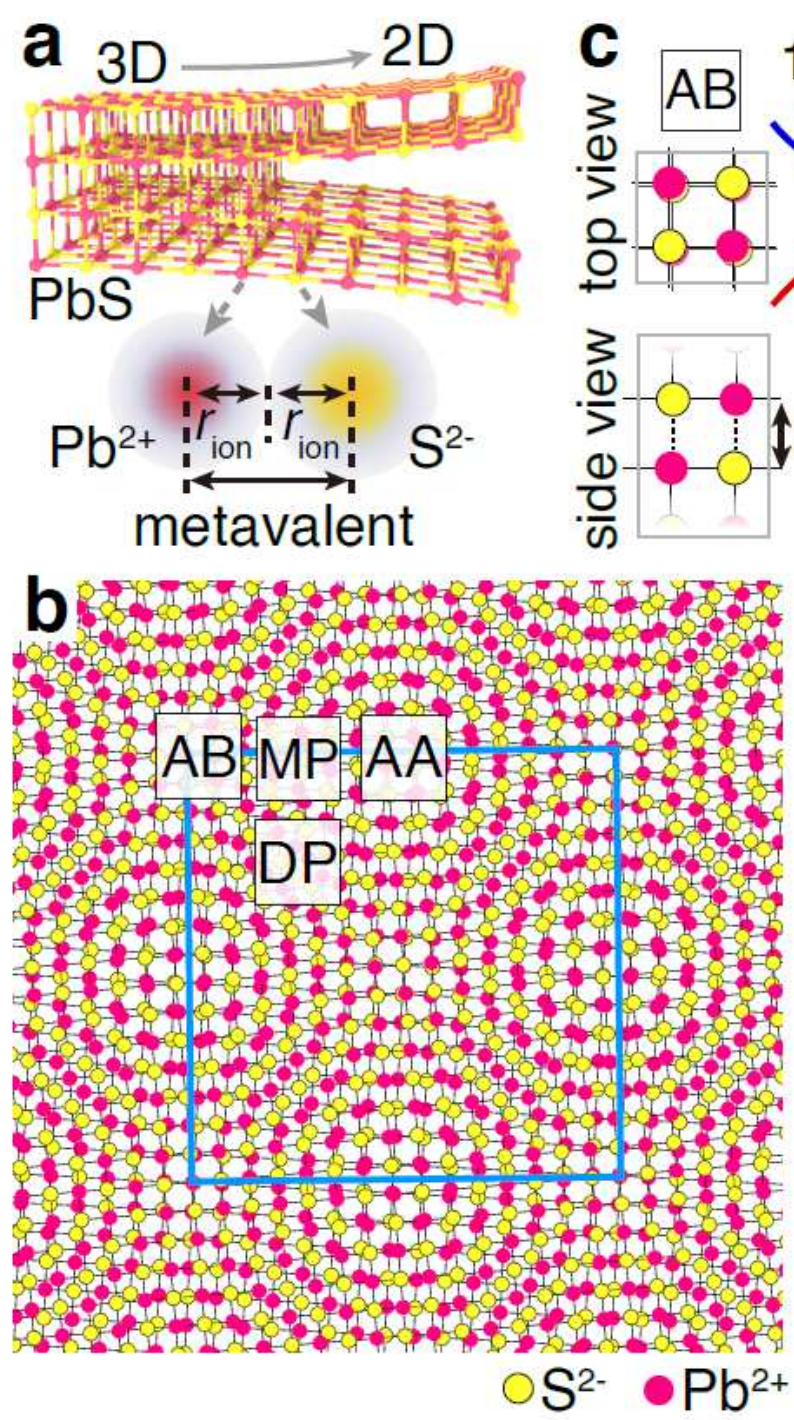
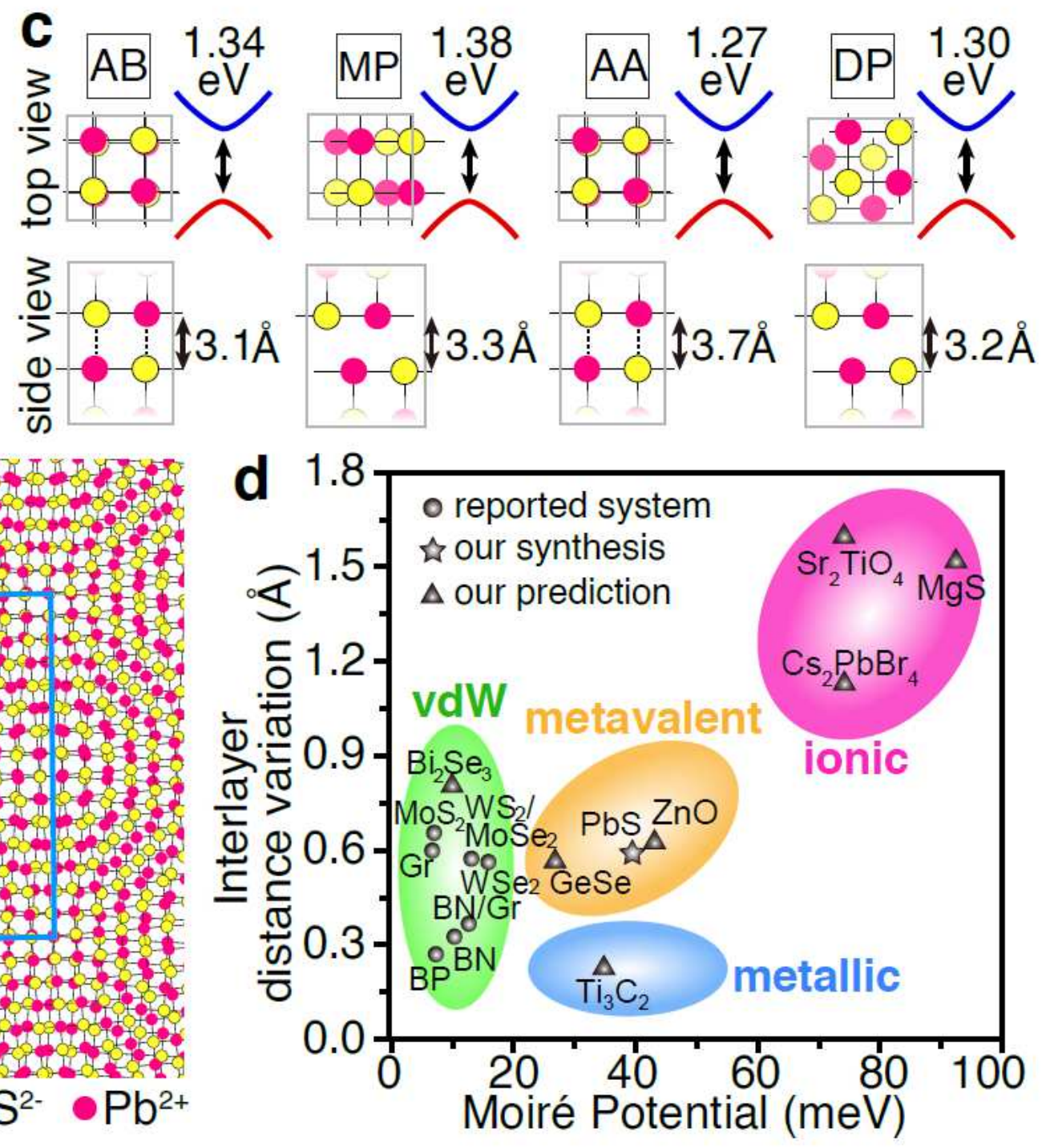

Figure 1

Structure and strong coupling of PbS moire superlattice. a, 3D and the cleaved 2D structures of PbS rocksalt crystal, emphasizing the metavalent interaction in all directions. b, Different local atomic alignments occur in a PbS moire superlattice with a twist angle of $8 \mathrm{Q}$. Blue square marks the moire unit-cell. Four representative stacking configurations are highlighted as $\mathrm{Pb}$ on $\mathrm{Pb}(\mathrm{AA}), \mathrm{Pb}$ on $\mathrm{S}(\mathrm{AB})$, middle point (MP), and diagonal point (DP). c, DFT calculations of $A A, A B, M P$, and DP configurations, showing the structure, bandgap, and interfacial distance of each configuration. d, DFT calculations on varieties of moire superlattices, including the reported vdW superlattices, the metavalent PbS synthesized in this work, and our predictions of other chemically bonded superlattices. The results show their moire potential and the largest interfacial distance variation among different stacking configurations. 

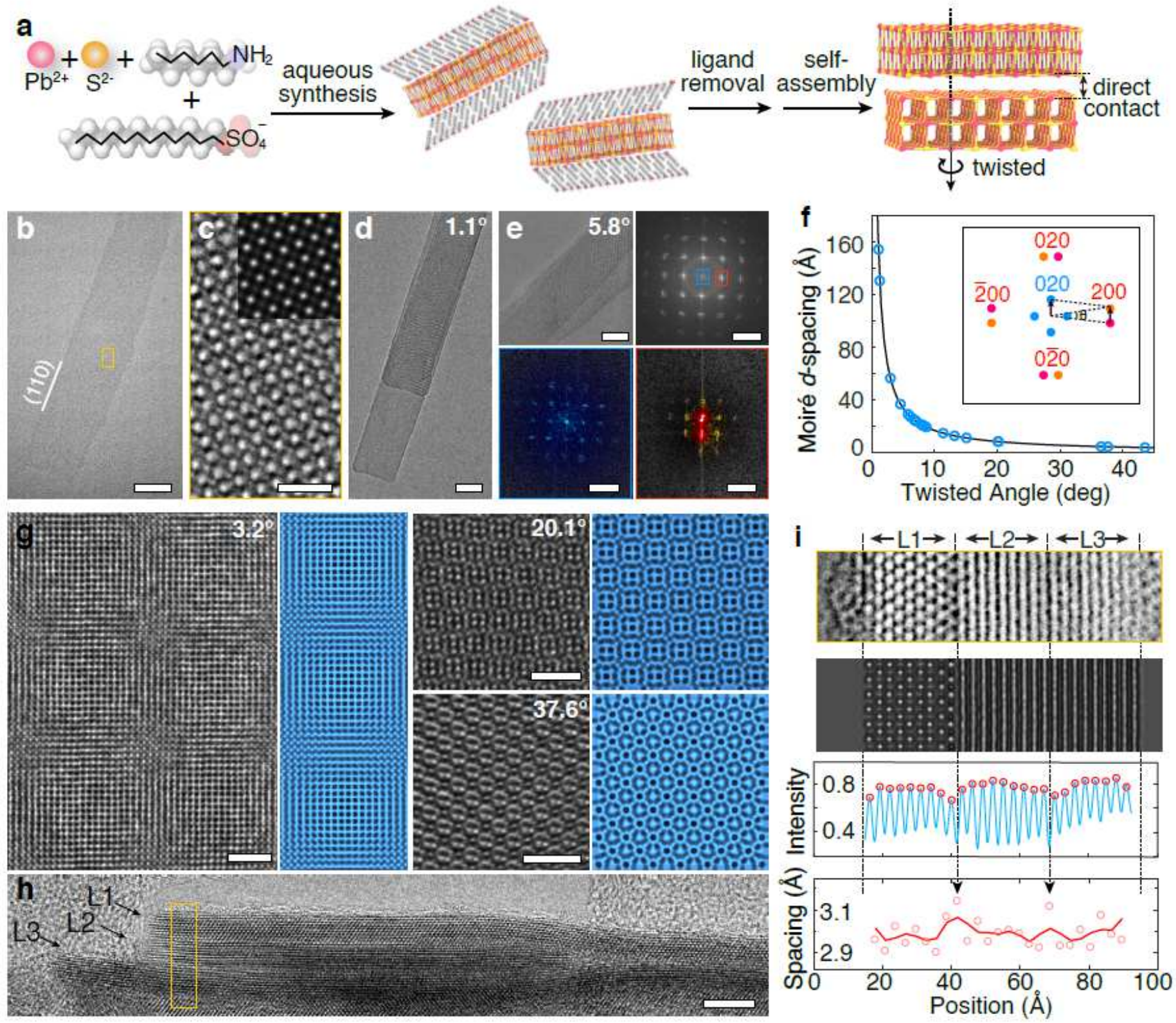

\section{Figure 2}

Synthesis and TEM characterizations of PbS moire' superlattices. a, Schematics of synthetic procedures. b, Low magnification TEM image of a single ultra-thin $\mathrm{PbS}$ nanosheet. c, Atomic resolution image of the boxed area in panel $b$ with an inserted simulation of TEM image. $d$, TEM image of a bilayer moire superlattice with a 1.1区 twist angle. e, TEM image (top-left) and FFT pattern (top-right) of a bilayer moire superlattice with a 5.8囚 twist angle. Two bottom panels show the enlarged details of the FFT pattern, in which blue dots indicate moire spatial frequencies, red dots indicate the (200) spatial frequencies of two individual rock-salt nanosheets, and orange dots indicate the emerged pattern from moire pattern and individual rock-salt patterns. f, Theoretical (black curve) and observed (green hollow dots) relationship between moire d-spacing (200) and twist angles. Inset shows the relationship between moire spatial frequencies (green) and two sets of individual rock-salt spatial frequencies (red and orange). g, Atomic 
resolution TEM images and corresponding simulated images (false coloured) of bilayer moire superlattices with a variety of twist angles. h, Side view of a moire superlattice composed of three layers, L1, L2, and L3. The region marked by the orange box is flipped and zoomed in panel i. i, Structural analysis of side-view details. From top to bottom are original TEM image, simulated image, image intensity and peak position of each atomic layer, and each interlayer spacing. Scale bar: b, $30 \mathrm{~nm} ; \mathrm{c}, 1 \mathrm{~nm}$; d, 100 nm; e, 30 nm (top left), 5 nm-1 (top right), 0.5 nm-1 (bottom two); g, 2 nm; h, 5 nm.
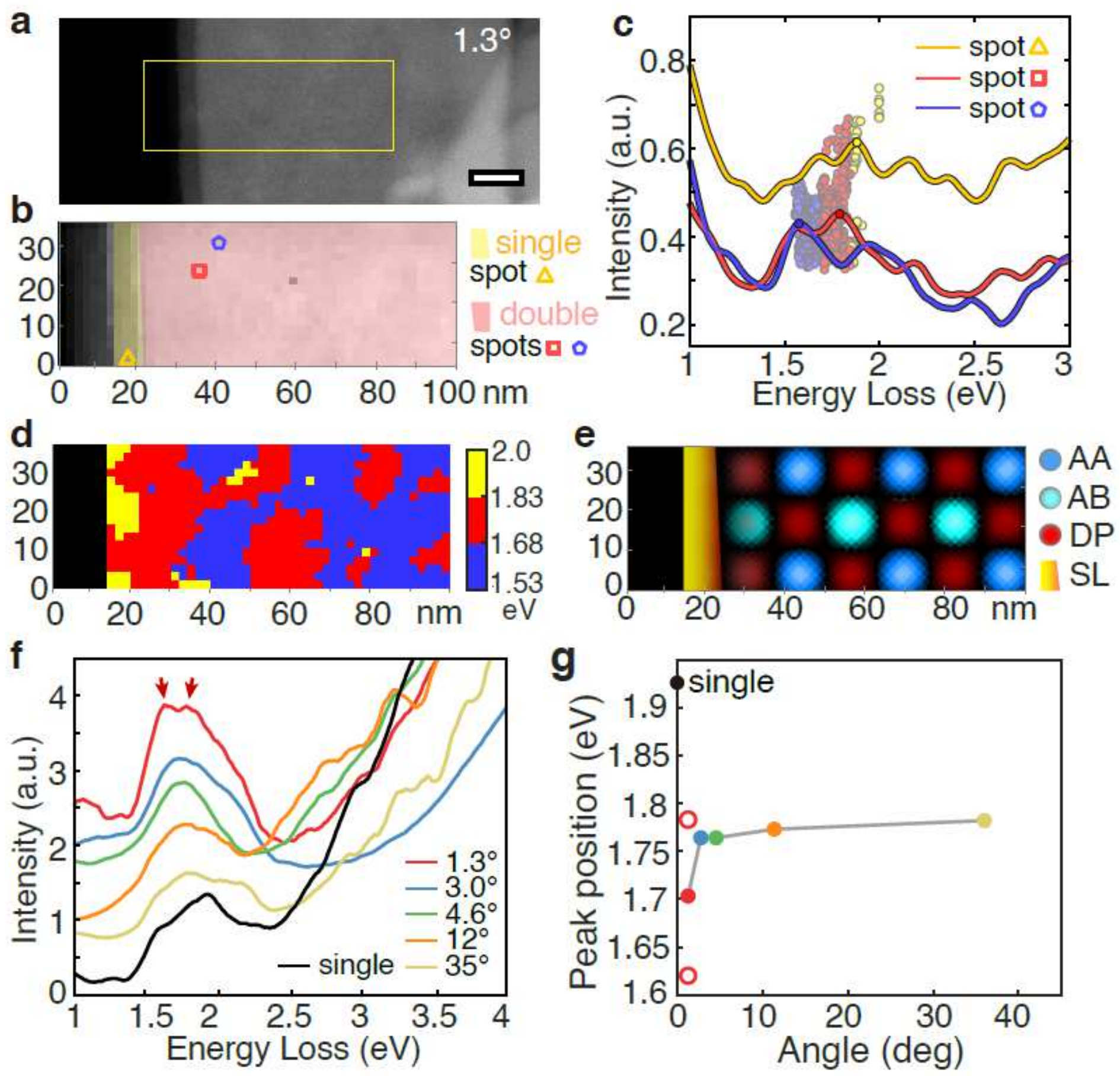

Figure 3 
Mapping of electronic states through STEM-EELS. a, Overview image of a moire superlattice with a twist angle of 1.3凶. Yellow box marks the scanning region for STEM-EELS. Scale bar, $20 \mathrm{~nm}$. b, Scanned image with single- and double-layer regions false-coloured and three representative spots marked. c, Plot of lowloss spectra from three representative spots with the spectral peaks marked with opaque dots. For other scanned spots, the spectra are omitted for clarity, only their spectral peaks are illustrated by the semitransparent dots. Dot colour follows the colourmap in $d$. $d$, Spatial mapping of the energy of spectral peaks that are shown by scattered dots in c. e, Simulated pattern of $A A, A B$, and DP configurations and single layer (SL) region in the moire superlattice with a twist angle of 1.3囚. f, Integrated spectra of the double-layer regions of moire superlattices with a variety of twist angles. A spectrum of single-layer PbS nanosheet is added for comparison. $\mathrm{g}$, The change of the peak energy of double-layer regions upon the change of the twist angle. Hollow dots at $1.3 \otimes$ shows the position of two separated peaks (marked by red arrows in panel f), whereas the solid dot shows the average of the two peaks. Energy dispersion of all EEL spectra is $9 \mathrm{meV} /$ pixel.
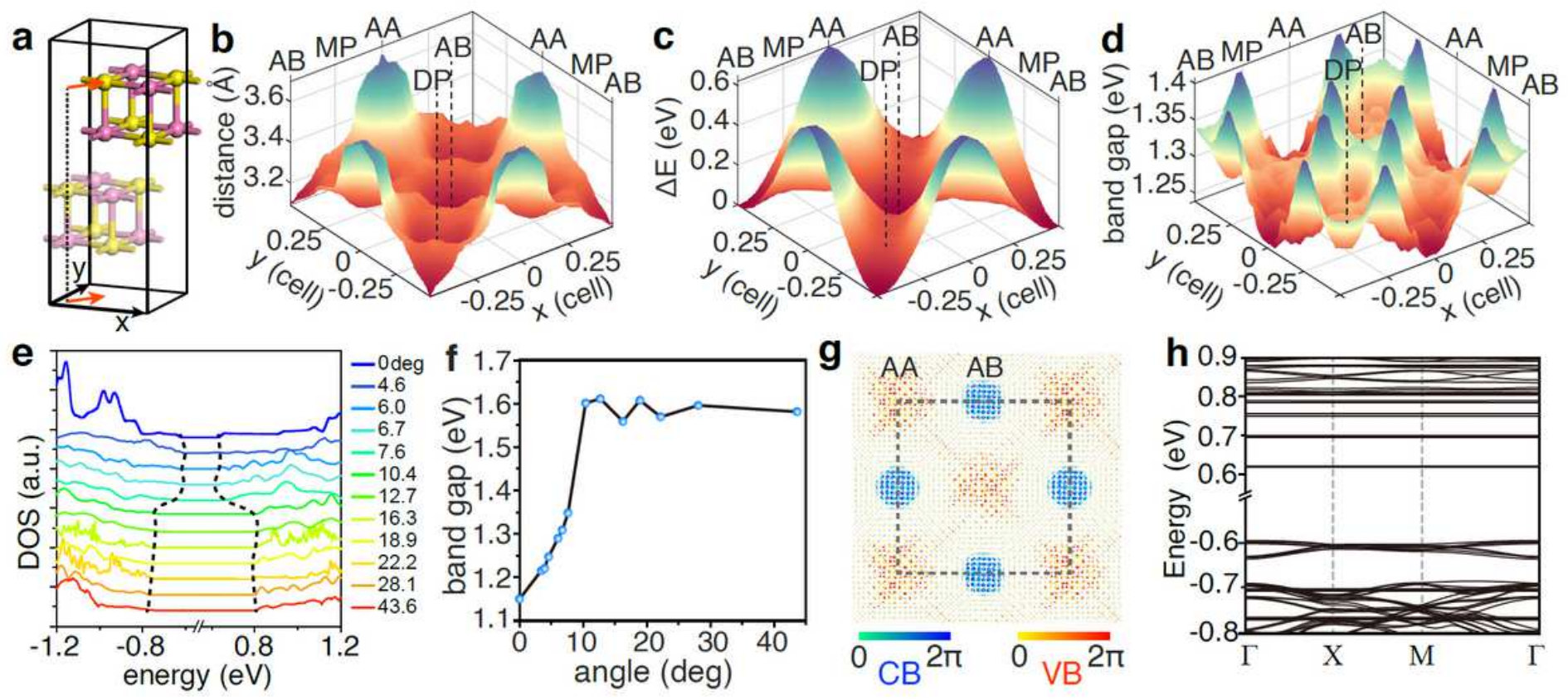

\section{Figure 4}

DFT calculations of the electronic localization. a, Illustration of shifting two PbS nanosheets for creating different stacking configurations and calculating its impact on the structural and electronic properties. b$d$, Change of the interfacial distance (b), free energy (c), and bandgap (d) upon the bilayer lateral shift. $x$ and $y$ are the fractional coordinates of the lateral shift. e, Direct calculation of the density-of-states of commensurate moire superlattices with a variety of twist angles. Black dashed lines indicate the positions of first valence and conduction bands. $\mathrm{f}$, Bandgap change upon twist angles extracted from panel e. g, Charge density of valence band (CB) and conduction band (VB). Colour is coded by the phase of wave function. $h$, Band structure of the moire superlattice with a $3.47 \otimes$ twist angle.

\section{Supplementary Files}


This is a list of supplementary files associated with this preprint. Click to download.

- PbSsuplnfo.pdf 